ACCEPTED MANUSCRIPT

\title{
Predicting bio-inspired candidate surfaces with superomniphobic characteristics
}

To cite this article before publication: Nikolaos Lempesis et al 2020 Surf. Topogr.: Metrol. Prop. in press https://doi.org/10.1088/2051$\underline{672 X / a b 9419}$

\author{
Manuscript version: Accepted Manuscript \\ Accepted Manuscript is "the version of the article accepted for publication including all changes made as a result of the peer review process, \\ and which may also include the addition to the article by IOP Publishing of a header, an article ID, a cover sheet and/or an 'Accepted \\ Manuscript' watermark, but excluding any other editing, typesetting or other changes made by IOP Publishing and/or its licensors" \\ This Accepted Manuscript is @ 2020 IOP Publishing Ltd.
}

During the embargo period (the 12 month period from the publication of the Version of Record of this article), the Accepted Manuscript is fully protected by copyright and cannot be reused or reposted elsewhere.

As the Version of Record of this article is going to be / has been published on a subscription basis, this Accepted Manuscript is available for reuse under a CC BY-NC-ND 3.0 licence after the 12 month embargo period.

After the embargo period, everyone is permitted to use copy and redistribute this article for non-commercial purposes only, provided that they adhere to all the terms of the licence https://creativecommons.org/licences/by-nc-nd/3.0

Although reasonable endeavours have been taken to obtain all necessary permissions from third parties to include their copyrighted content within this article, their full citation and copyright line may not be present in this Accepted Manuscript version. Before using any content from this article, please refer to the Version of Record on IOPscience once published for full citation and copyright details, as permissions will likely be required. All third party content is fully copyright protected, unless specifically stated otherwise in the figure caption in the Version of Record.

View the article online for updates and enhancements. 


\title{
Predicting bio-inspired candidate surfaces with superomniphobic characteristics
}

\author{
Nikolaos Lempesis ${ }^{1,2, a)}$, Aleš Janka ${ }^{3}$, Oksana Gnatiuk ${ }^{4}$, Stef J. L. van Eijndhoven ${ }^{4}$, Rudolf J. \\ Koopmans $^{1,2}$ \\ ${ }^{1}$ Plastics Innovation Competence Center, Passage du Cardinal 1, Fribourg CH-1700, Switzerland, \\ ${ }^{2}$ Department of Mechanical Engineering, College of Engineering and Architecture Fribourg HES- \\ SO, Bd de Pérolles 80, Fribourg CH-1705, Switzerland \\ ${ }^{3}$ Institute of Complex Systems, College of Engineering and Architecture Fribourg HES-SO, Bd de \\ Pérolles 80, Fribourg CH-1705, Switzerland \\ ${ }^{4}$ Department of Mathematics and Computer Science, Eindhoven University of Technology, \\ Eindhoven $5600 \mathrm{MB}$, the Netherlands

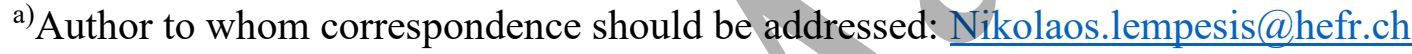

\begin{abstract}
We report the development and application of a refined version of the classical CassieBaxter wetting model for the prediction of surface topographies with superomniphobic traits. The sagging height defined through the capillary length was utilized to assess the relation between a curved liquid-air interface and the surface texture. The wettability, expressed in terms of the static apparent contact angle, was quantified for single- and double-scale surface topographies and for three representative liquids and the results were compared to those of the classical Cassie-Baxter model. Of the three single-scale topographies considered in this work, the fiber case exhibited the highest contact angle across length scales of surface topographies, whereas decreasing the length scale of surface patterns from a few hundreds of micrometers to a few hundreds of nanometers led to contact angle increase by $15-20 \%$. A generic expression for modeling multiscale hierarchical roughness of arbitrarily large multiplicity $n$ was derived and applied. Multiscale hierarchical roughness was corroborated to be a promising way for achieving enhanced liquid repellency. Double-scale roughness was more efficient when the two length scales differed in size by at least one order of magnitude. The "fiber on sinusoid" hierarchical topography exhibiting re-entrant geometry yielded contact angles over $150^{\circ}$ for all considered wetting liquids.
\end{abstract}




\section{INTRODUCTION}

A great amount of consumable commodities of daily life, such as food, water and other edible domestic products is lost due to the adhesion of these goods to their packaging material.[1] Development of new and refinement of existing technologies for manufacturing innovative packaging materials that repel all kinds of liquids and thereby reduce the product loss become increasingly significant. Besides the obvious product-loss problem, adhesion of food to packaging materials is directly associated with poor product appearance[2] and increased package recycling costs.[3] Moreover, in industrial plants, product adhesion to machinery is responsible for increased surface-cleaning costs. Therein, two significant side effects of microscopic soiling of plant equipment are related to sanitary problems[4] and production deterioration, both in terms of quality and quantity, due to fouling.

Despite the numerous different theories trying to explain and quantify adhesion of materials, stemming from various fields of knowledge, such as mechanics,[5, 6] thermodynamics,[7] physics[8,9] and chemistry,[10] up to now, there is no generic adhesion relationship applicable to all cases. However, surface wettability was identified as the most prevalent mechanism associated with adhesion.[3] The wettability refers to the interaction between a liquid and a solid surface and can be defined either as the ability of the surface to promote (large wettability) or withstand (small wettability) imbibition by a supernatant liquid, or, equivalently, as the tendency of a liquid to spread on (large wettability) or adhere to (small wettability) the solid surface. In the literature, both definitions are equivalent and complementary, depending on whether the reference point is the surface or the liquid. Throughout this work, both definitions of wettability were used interchangeably.

The apparent contact angle $\theta$, also referred to as the static apparent contact angle to emphasize that the liquid droplet sits at rest (at equilibrium) on top of the surface, is used in the literature extensively as a measure of wettability.[11] The larger the apparent contact angle (henceforth contact angle (CA)), the smaller the wettability and vice versa. Angle $\theta$ is defined as the angle formed between the solid surface and the tangent to the liquid-air interface at the contact point of all three phases.[12] In fact, for three-dimensional representations, this contact point constitutes a curve, the so-called triple-phase contact curve, along which all three phases come in contact. Without any loss of generality and for simplicity, the wetting systems (surfaces and droplets) are 
typically studied in a two-dimensional (2D) projection, which is then extended to infinity along the remaining dimension to form semi-infinite three-dimensional (3D) representations. Usage of $2 \mathrm{D}$ representations in wettability models, albeit simplified, was shown to be in qualitative agreement with realistic 3D measurements.[13, 14]

By convention,[15] when the contact angle is larger than $90^{\circ}$, the surface is characterized as phobic to the wetting liquid and repulsion is stronger than attraction between/surface and supernatant liquid. In the opposite case $\left(\theta<90^{\circ}\right)$, the surface is regarded as philic to the liquid and wetting occurs.[16] Based on this definition, water-repelling surfaces are called hydrophobic, oilrepelling surfaces are called oleophobic and surfaces that repel all kinds of liquids are identified as omniphobic. When the contact angle between an omniphobic surface and a liquid becomes larger than $150^{\circ}$, the surface is then called superomniphobic.

The first modeling attempt to describe the wettability of a surface was initiated over two hundred years ago, in 1805, by the British mathematician Thomas Young.[12] Young proposed a relatively simple relation to describe the force balance of a system at mechanical equilibrium: $\cos \theta^{\mathrm{Y}}=\left(\gamma_{\mathrm{sa}}-\gamma_{\mathrm{sl}}\right) / \gamma_{\mathrm{la}}$, where $\gamma_{\mathrm{sa}}$ is the surface energy of the solid surface, $\gamma_{\mathrm{la}}$ is the surface tension of the liquid, $\gamma_{\mathrm{sl}}$ is the interfacial energy at the interface between solid and liquid and $\theta^{\mathrm{Y}}$ denotes the uniquely defined[17] contact angle, often referred to as the equilibrium contact angle, which is prescribed by the chemical nature of the solid and the supernatant liquid.[18] Superscript Y denotes calculation of the contact angle by Young's relation. Young's equation, albeit rigorous and robust, is based on the rather simplistic assumption that the solid surface is completely flat and smooth (ideal surface), whereas full wetting is assumed, i.e., the wetting liquid is in full contact with the surface at all points and, therefore, no air is trapped between the liquid droplet and the surface.

Nonetheless, Young's equation was used exclusively for more than one hundred and twenty years until Wenzel[19] suggested in 1936 a refinement of Young's original model by introducing the notion of surface roughness $r$ into the calculation of the contact angle: $\cos \theta^{\mathrm{W}}=r \cos \theta^{\mathrm{Y}}$, where the superscript $W$ denotes calculation of the contact angle by virtue of Wenzel's relation. The roughness $r$ is defined as the quotient of the actual area of the rough surface over the total projected area. It follows, that for a rough surface $r>1$, whereas $r=1$ corresponds to a perfectly smooth surface. This relation leads to the following inequalities: $\cos \theta^{\mathrm{W}} \geq \cos \theta^{\mathrm{Y}}$ for $\theta^{Y}<90^{\circ}$ (philic surface) and $\cos \theta^{\mathrm{W}} \leq \cos \theta^{\mathrm{Y}}$ for $\theta^{Y}>90^{\circ}$ (phobic surface), which can be interpreted in the following way: the introduction of roughness, expressed by $\theta^{\mathrm{W}}$, to an originally ideal surface enhances the philic 
or phobic nature of the original surface. In other words, the introduction of surface roughness makes an originally philic flat surface $\left(\theta^{Y}<90^{\circ}\right)$ even more philic $\left(\theta^{W}<\theta^{\mathrm{Y}}<90^{\circ}\right)$ or, similarly, an originally phobic flat surface $\left(\theta^{Y}>90^{\circ}\right)$ even more phobic $\left(\theta^{W}>\theta^{\mathrm{Y}}>90^{\circ}\right)$.

The consideration of roughness by Wenzel brought about a big step towards finding a generic model describing realistic surface textures. However, like in the Young model, also in the Wenzel model, full wetting was assumed. This assumption is unrealistic and becomes true only when the distance between neighboring surface irregularities increases significantly and thus, the surface topography approaches that of a completely flat and smooth surface. In any other case, the surface microstructure, comprising crevices, cusps and indentations, results in partial wetting, expressed by the formation of air pockets between the supernatant liquid and the rough surface.

For the first time, in 1944, Cassie and Baxter[20] suggested a model that takes into consideration partial wetting of a rough surface: $\cos \theta^{\mathrm{CB}}=f_{\mathrm{sl}} \cos \theta^{\mathrm{Y}}-f_{\text {la }}$, where $f_{\text {sl }}$ and $f_{\text {la }}$ describe the area fractions of the solid-liquid and liquid-air interfaces, respectively, and the superscript CB denotes the calculation of the $\mathrm{CA}$ by virtue of the Cassie-Baxter $(\mathrm{CB})$ relation. The relationships for the calculation of $f_{\mathrm{sl}}$ and $f_{\text {la }}$ are as follows:

$f_{\mathrm{sl}}=\frac{\text { actual wetted area }}{\text { total projected area }}$

$f_{\mathrm{la}}=\frac{\text { actual nonwetted area }}{\text { total projected area }}$

By observing equations (1) and (2), it becomes obvious that the calculation of $f_{\mathrm{sl}}$ and $f_{\mathrm{la}}$ depends greatly on the surface topography. For the special case where $f_{\text {la }}=0$ and $f_{\mathrm{sl}}=1$, the CB model reduces to the Young model. The relation $f_{\mathrm{la}}=0$ indicates that no liquid-air interface exists and, therefore, full wetting occurs, whereas the relation $f_{\mathrm{sl}}=1$ is satisfied only when, in accordance with equation $(1)$, (actual wetted area) $\approx$ (total projected area). This condition holds when the surface roughness decreases and, eyentually, disappears completely and the surface texture resembles that of an ideal smooth surface (Young state).

It is well established in the literature that wettability is a synergistic result, influenced by chemical composition of the surface, multiscale roughness, overall topography, and possibly other factors related to the dynamic nature of the surface.[21] In some cases, modifications of tunable parameters such as temperature,[22] mechanical loading,[23] crystallization time[24] and applied electric field[25, 26] allowed (ir)reversible switching between superhydrophilicity and 
superhydrophobicity. Theoretically, the wettability of a surface can be enhanced or suppressed by engineering and tailoring all these parameters. As a first step towards quantifying the impact of all these contributions to nonwetting behavior, researchers focused on studying them separately. To this end, numerous works[27-31] considered changes solely in the chemical composition of the surface in order to reduce the surface energy $\gamma_{\text {sa. }}$. Based on Young's model, reduction of the surface energy leads to the formation of large contact angles and, thus, enhancement of the phobic character of the surface. However, chemistry modifications of the surface had certain limitations. For instance, the maximum contact angle of water on a flat $-\mathrm{CF}_{3}$ - terminated surface, which was reported to have the lowest surface energy, was found to be about $120^{\circ}$.[32] It becomes, therefore, obvious that alternative and more effective means need to be considered for more enhanced nonwetting behavior.

A more promising way to manipulate the wettability of surfaces is by changing the surface topography. It has been demonstrated in the past, that changes in the details of the local texture of a surface have a major influence on water repellency of hydrophobic surfaces.[33] Accordingly, superhydrophobic surfaces were obtained after changing the surface topography by various processing methods including deposition of gold clusters,[34] phase separation of polymer blends,[35] lithography,[36] plasma fluorination of a polymer surface,[37, 38] usage of densely packed aligned carbon nanotubes,[39] usage of aligned polyacrylonitrile nanofibers,[39] to name a few. Of particular relevance to that, surface roughness manipulation was proven to be very promising even in the design of superoleophobic surfaces.[40] Oil-repellent materials are very rare and difficult to synthesize because oils have very low surface tension $\gamma_{\text {la }}$ and hence exhibit increased tendency to spread.[30] Tuteja et al.[41] highlighted that the creation of highly nonwetting surfaces requires $\theta^{\mathrm{Y}}>90^{\circ}$, however, to date, there are no reports of a natural or artificial surface with a low enough surface energy $\gamma_{\mathrm{sa}}$ to enable $\theta^{\mathrm{Y}}>90^{\circ}$. The difficulty of finding surfaces exhibiting $\theta^{\mathrm{Y}}>90^{\circ}$ becomes even more pronounced when the surface is in contact with low surface tension liquids (e.g., alkanes).[40]

Interestingly, extensive studies on natural biological surfaces, such as leaves of plants Cotinus coggygria, Ginkgo biloba[42] and Nelumbo nucifera[43] revealed the possibility of designing surface textures with surprisingly high nonwetting capacities even though $\theta^{\mathrm{Y}}<90^{\circ}$. The superhydrophobic behavior of these textured surfaces was attributed to them being in a metastable state rather than in a stable equilibrium state. Indeed, in the seminal works of Marmur[44] and 
Nosonovsky,[45] thermodynamic considerations were employed to determine whether particular textured surfaces existed in a metastable or stable state. A few years later, Gibbs surface energy minimization in various solid-liquid-air systems showed that the Wenzel and Cassie-Baxter states can indeed be metastable and that such metastable states can be very long-lived.[46] In light of this evidence, one can claim that it is possible to design superomniphobic surfaces even though we are limited to materials exhibiting $\theta^{\mathrm{Y}}<90^{\circ}$.

At this point, a clarification regarding surface creation is in order. In the process of manufacturing superomniphobic surfaces, it's important to note that biological surfaces are, in general, different from engineered ones due to the hierarchical organization that characterizes the former.[47] By hierarchical organization, we mean that there are at least two levels of rough structures placed on top of each other. A typical example is the existence of microscale bumps (papillae) and nanoscale asperities placed upon these bumps in Nelumbo nucifera (lotus) leaves.[47] Hierarchical micro- and nanostructures induced increased surface roughness, as the surface-to-volume ratio grew for surfaces with two or more levels of roughness. Roughness at two or more levels was identified as the main cause of increased liquid repellency and surface phobicity.[48-50] To substantiate this observation, two explanations were suggested related to a) the kinetics of the droplet movement (not applicable here because only sessile drops are considered) and b) the thermodynamics of wetting. [51] Both these explanations converged to the conclusion that double-scale surface texture increased phobicity because it increased the surface roughness and eliminated contact angle hysteresis.[51] Therefore, the following key question arises: "which feature has greater impact on wettability? Hierarchical structure or roughness?"

Experimental studies revealed that surfaces with only one scale of roughness repelled well macroscopic droplets (e.g., rain droplets), however, they could not repel microscopic droplets (e.g., fog droplets) which were trapped easily between the coarse-scale surface irregularities,[47] thereby stressing the importance of hierarchical structure. Moreover, multiscale roughness was responsible for mechanically stable and durable liquid-repellent surfaces. This is due to the protection against mechanical damage and wear of the smaller scale (nanoscale) features provided by the larger scale (microscale) features.[52] In contrast, experiments with non-hierarchical patterned surfaces, which, however, retained some degree of roughness, demonstrated superhydrophobicity.[53] Additionally, nonwettable surfaces were produced successfully without necessarily employing multiscale roughness. [54, 55] These observations indicated that roughness 
was more important than hierarchical structure (multiscale roughness), the latter being, however, beneficial. Furthermore, roughness was found to have even greater impact on wettability than low surface energy $\gamma_{\mathrm{sa}}[$ [47]

For even more pronounced control over wettability, very recently, topography manipulation was combined successfully with chemical treatment of the surface to enhance/suppress wettability depending on technological application. Self-cleaning, strain-resistant and anti-bacterial superhydrophobic cotton fabric was produced for oil-water separation and applications requiring anti-bacterial capabilities.[56] In line with that, a bioinspired cotton for efficient separation of immiscible oil-water mixtures and emulsions was created to circumvent inherent difficulties of traditional mesh-based methods pertaining to penetration of microdroplets through the mess pores.[57] Similar technologies were applied to different substrate materials including steel[58] and synthetic leather[59] to promote green science and technology.[60] For an extensive overview of different substrate coatings and associated fabrication techniques the reader is referred to the comprehensive work of B. Bhushan and S. Martin.[61]

Engineering and eventually controlling wettability of a surface has attracted a lot of attention within the scientific community, in large part, due to the very broad spectrum of engineering applications associated with wetting. Numerous technological applications related to non-wetting textiles,[62, 63] anti-fogging,[64] anti-icing,[65] buoyancy,[66] flow improvement,[67] antibiofouling[68] and water collection from fog[69] have seen the light of day in the recent years. Most of these applications were inspired by the behavior of biological surfaces when interacting with various liquids. Examples vary from traditional applications of non-reflective surfaces utilizing the "moth-eye" effect,[70] surfaces for high and adjustable adhesion exploiting the "gecko-effect",[71] self-cleaning windows considering the self-cleaning mechanism or the socalled "lotus-effect",[72] water-repellent rough surfaces inspired by the water strider leg[73] and the shark skin[74] structure, to emerging applications related to energy conversion and conservation.[75]

The great technological potential related to predicting and controlling surface wettability is expressed by the great number of modeling works generated over the past decades.[11, 17, 41, 45, $47,75,76][46,77-82]$ The objective of the present work is to elaborate further on the theoretical understanding of the impact of multiscale roughness on surface wettability. We proceeded by studying the classical $\mathrm{CB}$ wetting model and subsequently suggesting a more realistic version of 
this model. The accuracy of the classical CB model has been questioned in the past.[83] In general, the area of validity of the Wenzel and CB equations is for uniformly rough surfaces.[84] It has been argued that for non-uniform rough surfaces or when the size of the surface heterogeneities was very small (of atomic or molecular dimensions), the original CB equation should be replaced by generalized expressions as extracted by means of surface energy minimization. $[85,86]$ Moreover, the original CB relation failed to predict the contact angle hysteresis for droplets under motion.[17]

\section{MATERIALS AND METHODSS}

Here, three different types of surface topographies were selected after carefully considering the points raised by Dufour et al.[87] who used the interface displacement theory[88] to study the wetting behavior of Gaussian and non-Gaussian random surfaces. These are: a) flat-top pillars, b) fibers with circular cross-section and c) sine waves. The shape and geometric details of each surface topography are illustrated in figure 1. As explained in the previous section, twodimensional projections of the semi-infinite 3D representations shown in figure 1 were considered in all subsequent analysis. For every surface topography, a set of geometric parameters $a, l$ and $l_{0}$ were used to fully represent its geometrical characteristics. The corresponding parameters are shown in figure 1 and summarized in Table 1. Parameter $l_{0}$ is used as input to define the length scale of interest. Specifically, for the pillar topography, where three parameters are required for its full description (see figure 1), $l_{0}$ was taken to be equal to the height of the pillars $H$. In the remaining two topographies, $l_{0}$ was varied from a few nanometers up to a few tens of micrometers.

These topographies were chosen because of their relative simplicity and because they approximate well known natural and artificial nonwetting surfaces. For instance, the sinusoidal topography mimics well the texture of the shark skin[89], whereas a hierarchical structure comprising fibers on top of sinusoidal pulses resembles the bumps on the Lotus leaf.[90] Finally, the flat-top pillar topography was used extensively in the literature[11, 46, 91] and serves as a reference system and point of comparison with previous studies. 
(B)
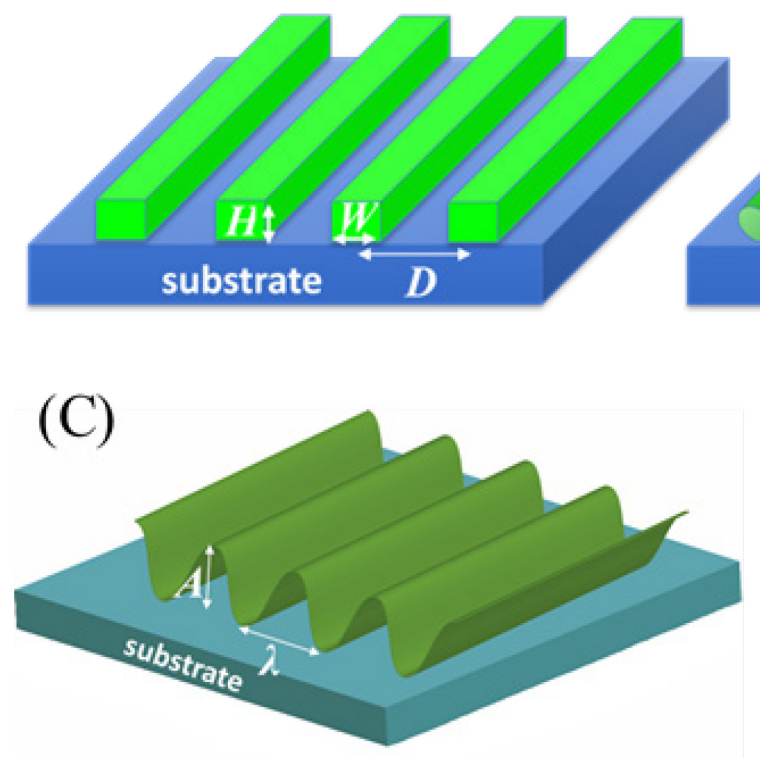

Figure 1. Three-dimensional illustrations of the three semi-infinite surface topographies considered here: A) flat-top pillars, B) fibers and C) sine wave. In all parts, the geometric parameters for the description of each topography are shown (see also Table 1).

Table 1. Geometric parameters for the description of the three single-scale surface topographies used in this work (see also figure 1).

\begin{tabular}{|c|c|c|c|}
\hline \multirow{2}{*}{ Surface topography } & \multicolumn{3}{|c|}{ Parameters } \\
\cline { 2 - 4 } & $a$ & $l$ & $l_{0}$ \\
\hline Flat-top pillars & pillar width $W$ & pillar distance $D$ & pillar height $H$ \\
\hline Fibers & fiber radius $R$ & fiber distance $D$ & varying \\
\hline Sinusoid & amplitude $A$ & wavelength $\lambda$ & varying \\
\hline
\end{tabular}

For all surface topographies, throughout this work, the surface material was linear low-density polyethylene (LLDPE) which constitutes a very common, well-characterized packaging material with ample experimental data in the literature. Because we aspire to help in the development of superomniphobic surfaces, in total three liquids were used in this work spanning the whole polarity spectrum: water (WAT), glycerol (GLY) and diiodomethane (DIM). Water is the most polar liquid 
of the three, followed by glycerol which retains moderate polarity due to the three -OH groups in its molecule and finally diiodomethane exhibiting only very limited polarity. These constitute commonly used liquids in accurate contact angle measurements.[92, 93] Table 2 summarizes the required physical properties of the three wetting liquids considered here. For more details on the derivation of the mathematical formulas presented next, the reader is referred to the supplementary material.

Table 2. Surface tension $\gamma_{\text {la }}$, Young's contact angle $\theta^{\mathrm{Y}}$ and density $\rho$ of the three wetting liquids considered here. In all cases, the surface material is LLDPE.

\begin{tabular}{|c|c|c|c|}
\hline Wetting liquid & $\boldsymbol{\gamma}_{\mathrm{la}}[\mathbf{m N} / \mathbf{m}]$ & $\boldsymbol{\theta}^{\mathbf{Y}}\left[{ }^{\mathbf{0}}\right]$ & $\boldsymbol{\rho}\left[\mathbf{k g} / \mathbf{m}^{\mathbf{3}}\right]$ \\
\hline WAT & 72.8 & 73 & 997 \\
\hline GLY & 64 & 76 & 1261 \\
\hline DIM & 50.8 & 57 & 3320 \\
\hline \multicolumn{2}{|r}{} \\
\hline
\end{tabular}

\subsection{Classical CB Model}

The classical CB model includes explicit formulas for the solid-liquid $f_{\mathrm{sl}}$ and liquid-air $f_{\mathrm{la}}$ area fractions (equations (1) and (2), respectively), whose calculation depends on the considered surface topography. In the calculation of these area fractions, a linear liquid-air interface was assumed. The resulting relations for the three considered single-scale surface topographies are as follows:

\subsubsection{Pillar Topography}

$$
f_{\mathrm{sl}}=\frac{W}{D}
$$

$f_{\mathrm{la}}=\frac{D-W}{D}=1-\frac{W}{D}$

Interestingly, in the context of the classical CB model, the wettability of surfaces featuring pillar patterns was independent of the pillar height $H$.

\subsubsection{Fiber Topography}

$f_{\mathrm{sl}}=\frac{2\left(\pi-\theta^{Y}\right) R}{D}$ 


$$
f_{\mathrm{la}}=1-2 \frac{R}{D} \sin \theta^{\mathrm{Y}}
$$

\subsubsection{Sinusoidal Topography}

$$
\begin{aligned}
& f_{\mathrm{sl}}=\left\{\begin{array}{l}
\frac{1}{\pi} \sqrt{1+(A k)^{2}}\left[E\left(\frac{A k}{\sqrt{1+(A k)^{2}}}\right)-E\left(\frac{A k}{\sqrt{1+(A k)^{2}}}, k x_{1}-\frac{\pi}{2}\right)\right], x_{1}>\frac{\lambda}{4} \\
\frac{1}{\pi} \sqrt{1+(A k)^{2}}\left[E\left(\frac{A k}{\sqrt{1+(A k)^{2}}}\right)+E\left(\frac{A k}{\sqrt{1+(A k)^{2}}}, k x_{1}-\frac{\pi}{2}\right)\right], x_{1}<\frac{\lambda}{4}
\end{array}\right. \\
& f_{\mathrm{la}}=\frac{1}{\pi} \arcsin \left[\frac{\tan \left(\pi-\theta^{Y}\right)}{A k}\right]
\end{aligned}
$$

where $k=2 \pi / \lambda$ is the wave number of the sinusoidal profile, $E(m)$ is the complete elliptic integral of the second kind, $E(m, v)$ is the incomplete elliptic integral of the second kind and $x_{1}$ is the $\mathrm{x}$ coordinate of the contact point calculated to be equal to $x_{1}=\frac{1}{k} \arcsin \left[\frac{\tan \left(\pi-\theta^{\mathrm{Y}}\right)}{A k}\right]$ (more details on this in section 1.3 of the supplementary material).

\subsection{Improved CB Model}

Most theoretical works making use of the classical CB model assumed that the liquid-air interface was a straight line. This simplifying assumption was justified by the typically small distance between neighboring surface irregularities present in micro- and nanotextured surfaces. Prominent exceptions to this consensus include the inspirational works of G. Carbone and L. Mangialardi[94] and F. Bottiglione and G. Carbone,[95] followed by two more recent publications[96, 97] where convex/concave-shaped liquid-air interfaces were considered. This work acts complementary to these works and examines how curved liquid-air interfaces in the form of menisci interact with different surface textures of increasing complexity.

Part of the novelty of this work lies in the definition of the radius of the liquid-air interface. In the aforementioned works, $[94,95]$ the radius of the curved liquid-air interface was defined as $r_{\text {la }}$ $=\gamma_{\mathrm{la}} / p$, where $p$ is the pressure difference across the liquid-air interface separating the drop from its external environment. However, this pressure difference was very difficult to be known a priori and therefore the radius of the curved liquid-air interface remained vaguely defined. Here, we 
adopted the more rigorous approach proposed by Tuteja et al.,[41] wherein the radius of the curved meniscus was defined with the help of the capillary length, which is a function of the liquid surface tension $\gamma_{\text {la }}$ and the density of the liquid $\rho$ (see below). We remind that in the original CB model, the involvement of the liquid characteristics was implicitly considered through the Young contact angle $\theta^{\mathrm{Y}}$, which depends on the chemical nature of the surface and the liquid. This approach did not require any prior knowledge of the pressure drop $p$ and did not suffer from contact boundaries issues arising during the interfacial energy minimization process.

As a first step, we revoked the intrinsic assumption of the classical CB model related to a linear liquid-air interface and introduced a curved liquid-air interface. This was done by considering the sagging height (or protrusion height)[41] $h$ and we proceeded by deriving new formulas for the solid-liquid and liquid-air area fractions that incorporated $h$. Based on Tuteja et al.,[41] the sagging height $h$ was defined by the following equation:

$$
h=\frac{l^{2}}{l_{\text {cap }}}
$$

where $l$ is a characteristic topography-dependent length and is given in Table 1 for each surface topography considered in this work. The capillary length[98] $l_{\text {cap }}$ was defined through equation (10):

$l_{\text {cap }}=\sqrt{\frac{\gamma_{l a}}{\rho g}}$

In equation (10), $g$ is the gravitational acceleration. Because the capillary length $l_{\text {cap }}$ depends on the surface tension of the liquid and the liquid density, it encloses the physicochemical characteristics of the wetting liquid thereby offering a richer representation of the liquid.

In the following sections, the derived expressions for the solid-liquid and liquid-air area fractions for the three surface profiles are presented for curved liquid-air interfaces due to sagging. The schematic representations of the corresponding geometries, as well as details on the derivation of these expressions are provided in the supplementary material. From equations (9) and (10), it is obvious that the sagging height depends on the liquid properties and the topography but not on gravity. Gravity and droplet size can indeed change the pressure in the drop (gravity only for the largest textures), however, in this work the effect of pressure on the capillary length and thus on the sagging height is neglected. Therefore, the effects of droplet size and weight have been neglected throughout this analysis, while droplets of spherical shape were assumed. Because this 
work focused primarily on sessile drops sitting at rest on a surface, consideration of the viscosity into the capillary length calculation was not thought relevant. Viscosity involvement is deemed more meaningful when droplet movement is involved and plays a role e.g., in predicting the selfcleaning behavior of a surface. Consequently, consideration of contact angle hysteresis, which is connected to droplet movement, remained out of the scope of the current work.

Nonetheless, in compliance with previous approaches, $[75,99,100]$ the revised solid-liquid area fractions $f_{\mathrm{s}}$ which were extracted here after considering the effect of sagging, constitute a measure of how easy a droplet can roll off a surface, providing thus insight into the self-cleaning behavior of a certain surface. In general, reduced solid-liquid area leads to reduced solid-liquid adhesion and thus to increased repellency and self-cleaning capacity. The wettability of surfaces by liquids under flow was studied extensively by employing finite volume simulations on the continuum scale,[101-104] where the dynamics and kinematics of the flow were the primary focus. This work acts complementarily to these efforts, tackling wettability from a different standpoint, namely focusing on surface optimization, rather than flow characterization.

\subsubsection{Pillar Topography}

For the pillar topography, we distinguished two different cases based on the outcome of one criterion. The criterion decided whether the liquid touched the base level of the surface. Figure 2 shows a schematic representation of the two distinct cases. For simplicity, it was assumed that in both cases the liquid was pinned at the corner points of the pillars, i.e., the triple-phase contact line was immobilized at the corner points of the pillars. The decision which one of the two cases prevailed hinged upon the geometrical characteristics of the pillar topography, as well as the sagging height $h$ which was in turn influenced by the wetting liquid and the geometrical characteristics (see equations (9) and (10)) of the surface. The characteristic length $l$ in equation (9) was taken to be equal to the pillar distance $D$, whereas parameter $l_{0}$, which was used as input and defined the length scale of interest, was equal to the pillar height $H$. Thus, for a given wetting liquid and fixed dimensions of the pillar topography, the established criterion identified which one of the two wetting scenarios was true. The sagging radius was equal to (see supplementary material):

$R_{\mathrm{sag}}=\frac{x^{2}+h^{2}}{2 h}$ 
where distance $x$ is equal to $x=(D-W) / 2$ and $h$ is the sagging height defined via equations (9) and (10). If the sagging height $h$ is smaller than the pillar height $H$, then the liquid does not touch the base level of the surface. If $h \geq H$, the liquid touches the base level of the surface. The resulting expressions of the area fractions for the two distinct cases are as follows.
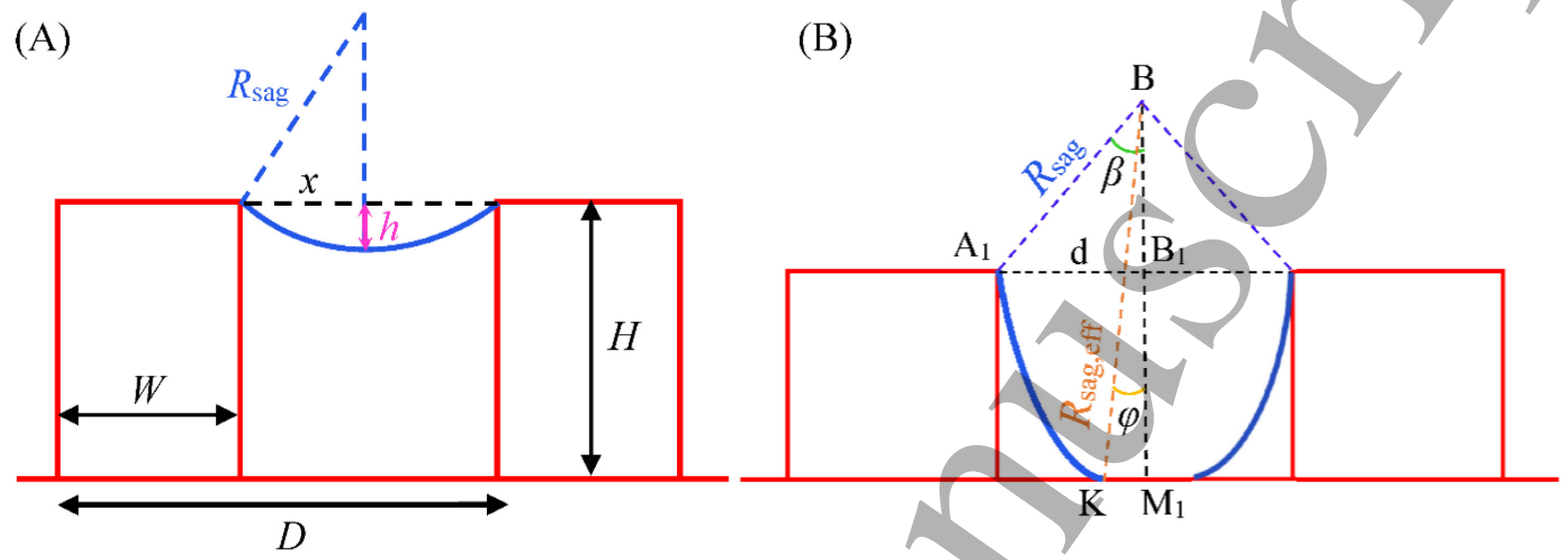

Figure 2. Schematic representation of the two cases for the pillar topography: A) one contact point $\&$ no touching and B) one contact point \& touching of the surface base level. In both parts, the surface is shown in red and the liquid-air interface with continuous blue line. The sagging radius $R_{\text {sag, }}$, the sagging height $h$, the distance $x$ and the geometric characteristics $(W, D, H)$ of the pillar topography, as well as angles $\varphi$ and $\beta$ are shown in parts (A) and (B) (see text for details).

\subsubsection{No Contact with the Surface Base Level}

When the liquid does not touch the surface base level [figure 2(a)], the area fractions are given by the following expressions:

$$
\begin{aligned}
& f_{\mathrm{sl}}=\frac{\text { actual wetted area }}{\text { total projected area }}=\frac{W}{D} \\
& f_{\mathrm{la}}=\frac{\text { actual nonwetted area }}{\text { total projected area }}=\frac{2 \beta R_{\mathrm{sag}}}{D}
\end{aligned}
$$

with

$$
\beta=\arcsin \left(\frac{D-W}{2 R_{\mathrm{sag}}}\right)
$$

\subsubsection{Contact with the Surface Base Level}


Sagged configurations such as the one depicted in figure 2(b) are, in general, unstable even in the case of a substrate with phobic $\theta^{\mathrm{Y}}$ exhibiting very limited lifetime, thereby resorting quickly to a fully wetted Wenzel state However, for purposes of completeness, these unstable configurations were considered and included in our analysis. In this case, the expressions for the area fractions are:

$f_{\mathrm{sl}}=\frac{\text { actual wetted area }}{\text { total projected area }}=\frac{W}{D}+\frac{2 R_{\mathrm{sag}}}{D} \sqrt{1-\cos ^{2} \varphi}$

Angle $\varphi$ is an auxiliary angle, whose calculation proceeded with equation (16):

$$
\begin{aligned}
& \cos \varphi=\frac{H+\sqrt{R_{\text {sag }}^{2}-d^{2}}}{R_{\text {sag }}} \\
& f_{\mathrm{la}}=\frac{\text { actual nonwetted area }}{\text { total projected area }}=\frac{2 R_{\text {sag }}(\beta-\varphi)}{D}
\end{aligned}
$$

The expression for the calculation of angle $\beta$ is identical to the one given in equation (14).

\subsubsection{Fiber Topography}

For the fiber profile, we distinguished four different cases based on two criteria. These cases are shown schematically in figure 3 . The first criterion decided whether we had one or two contact points. This was done by comparing the sagging radius $R_{\text {sag, }}$ calculated through equation (18), with a critical sagging radius $R_{\mathrm{sag}, 0}$, defined by equation (19):

$$
\begin{aligned}
& R_{\mathrm{sag}}=\frac{d^{2}+h^{2}}{2 h} \\
& R_{\mathrm{sag}, 0}=\frac{d}{\sin \theta^{\mathrm{Y}}}=\frac{D / 2-R \sin \theta^{\mathrm{Y}}}{\sin \theta^{\mathrm{Y}}}
\end{aligned}
$$

where $d=(D / 2)-R \sin \theta^{\mathrm{Y}}$

If $R_{\text {sag }} \geq R_{\mathrm{sag}, 0}$, then the droplet had a relatively large sagging radius and could not fit into the asperities of the fiber topography. As a result, there was only one contact point [figures 3(a) and 3(c)]. In the opposite case $\left(R_{\mathrm{sag}}<R_{\mathrm{sag}, 0}\right)$, the liquid had a relatively small sagging radius and penetrated further into the free space between adjacent fibers. This led to the formation of two contact points [figures 3(b) and 3(d)]. As in the case of pillars, the second criterion determined whether the wetting liquid touched the base level of the surface. It can be proven (see 
supplementary material) that the liquid touched the base level of the surface when $R\left(1-\cos \theta^{\mathrm{Y}}\right) \leq R_{\mathrm{sag}}-\sqrt{R_{\mathrm{sag}}^{2}-d^{2}}$. Parameter $l_{0}$, which was used as input and defined the length scale of interest, varied here, whereas the characteristic length $l$, which appears in equation (9) and influences the sagging height $h$, was the fiber distance $D$. As before (section 2.2.1.2), the "sagged" configurations depicted in figures 3(c) and 3(d) were of very limited lifetime and constituted rather unstable states. In that regard, once the liquid touched the surface base level, very rapidly, almost abruptly, the system transitioned to the more stable fully wetted Wenzel state. Although, these configurations constitute essentially transient states with minimal practical importance, for purposes of completeness, they were included in our analysis.

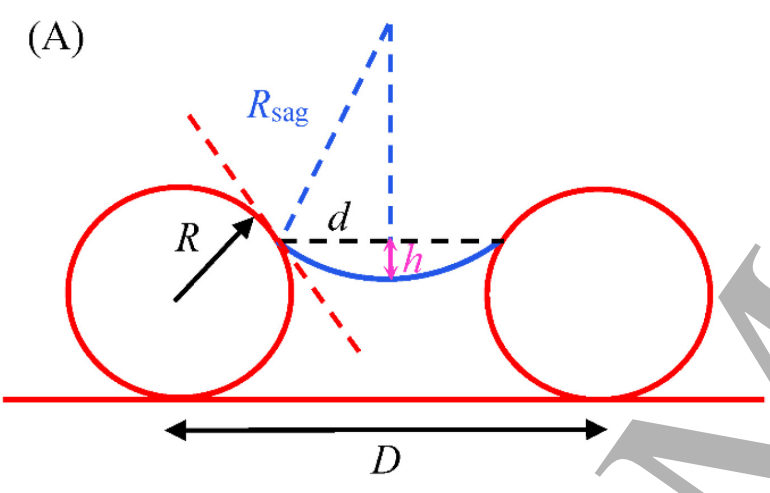

(C)

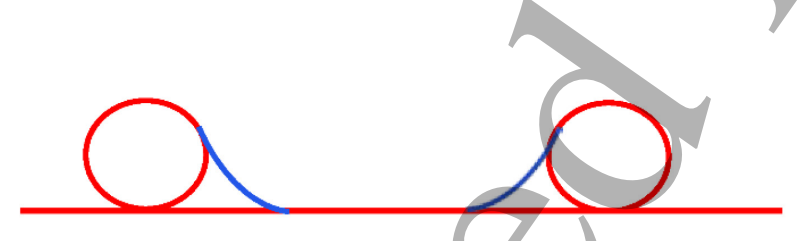

(B)

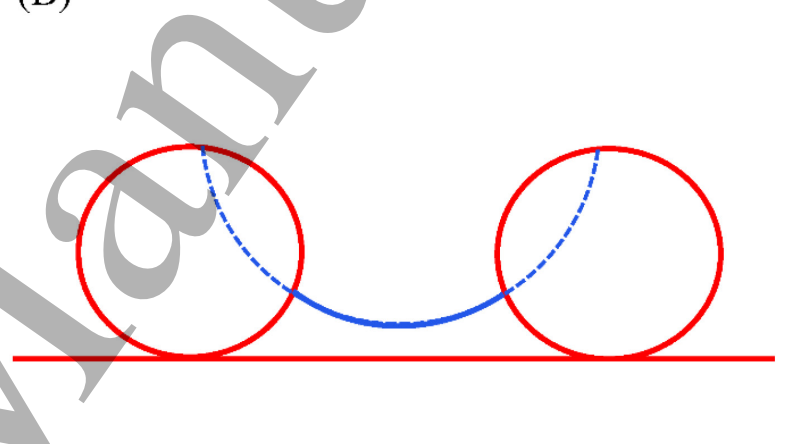

(D)

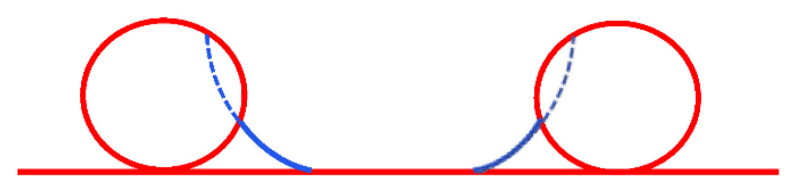

Figure 3. Schematic representation of the four cases considered for the fiber topography: A) one contact point \& no touching, B) two contact points \& no touching, C) one contact point \& touching of the surface base level and D) two contact points \& touching of the surface base level. In all parts, the surface is shown in red and the liquid-air interface with continuous blue lines. Broken blue lines outline the arch of an imaginary circle with the same center and radius as the liquid interface. The broken red line depicts the tangent to the fiber circumference at the contact point. The sagging radius $R_{\text {sag, }}$, the sagging height $h$, distance $d$ and the geometric characteristics $(R, D)$ of the fiber topography are shown in part (A) (see text for details). 


\subsubsection{One Contact Point \& No Touching of the Surface Base Level}

For this first case shown in figure 3(a), the corresponding expressions for the area fractions are:

$$
\begin{aligned}
& f_{\mathrm{sl}}=\frac{\text { actual wetted area }}{\text { total projected area }}=\frac{2 R\left(\pi-\theta^{\mathrm{Y}}\right)}{D} \\
& f_{\mathrm{la}}=\frac{\text { actual nonwetted area }}{\text { total projected area }}=\frac{R_{\mathrm{sag}} 2\left(\pi-\theta^{\mathrm{Y}}\right)}{D}
\end{aligned}
$$

\subsubsection{Two Contact Points \& No Touching of the Surface Base Level}

This case is shown schematically in figure $3(\mathrm{~b})$. The area fractions are as follows:

$f_{\mathrm{sl}}=\frac{\text { actual wetted area }}{\text { total projected area }}=\frac{2 R\left(\pi-\theta^{\mathrm{Y}}\right)}{D}+\frac{2 R\left(\theta^{\mathrm{Y}}-\frac{\pi}{2}-\gamma\right)}{D}$

where angle $\gamma$ is a solution to the equation $\tan ^{2}\left(\frac{\gamma}{2}\right)-G \tan \left(\frac{\gamma}{2}\right)+F=0$ and $G, F$ are parameters defined and calculated in the supplementary material,

$$
f_{\mathrm{la}}=\frac{2 \beta R_{\text {sag,eff }}}{D}=\frac{2 R_{\text {sag,eff }}}{D} \arccos \left[\frac{\left(\sqrt{R_{\text {sag }, 0}^{2}-d^{2}}-R \cos \theta^{\mathrm{Y}}-R \sin \gamma\right)}{R_{\text {sag,eff }}}\right]
$$

\subsubsection{One Contact Point \& Touching of the Surface Base Level}

This case is illustrated in figure 3(c). The area fractions for this case are:

$$
f_{\mathrm{sl}}=\frac{\text { actual wetted area }}{\text { total projected area }}=\frac{2 R\left(\pi-\theta^{\mathrm{Y}}\right)}{D}+\frac{2 R_{\mathrm{sag}}}{D} \sqrt{1-\cos ^{2} \varphi}
$$

where angle $\varphi$ is calculated through equation (25):

$$
\begin{aligned}
& \cos \varphi=\frac{R}{R_{\mathrm{sag}}}\left[1-\cos \theta^{\mathrm{Y}}+\frac{R_{\mathrm{sag}}}{R} \sqrt{1-\left(\frac{d}{R_{\mathrm{sag}}}\right)^{2}}\right] \\
& f_{\mathrm{la}}=\frac{\text { actual nonwetted area }}{\text { total projected area }}=\frac{2 R_{\mathrm{sag}}}{D}(\beta-\varphi)
\end{aligned}
$$

where angle $\beta=\arcsin \left(d / R_{\text {sag }}\right)$. 


\subsubsection{Two Contact Points \& Touching of the Surface Base Level}

This case constitutes a combination of the two previous cases and is schematically represented in figure 3(d). Similar to the previous case, this wetting configuration was rather unstable and was added to our analysis for the sake of completeness. The solid-liquid area fraction is equal to:

$$
f_{\mathrm{sl}}=\frac{\text { actual wetted area }}{\text { total projected area }}=\frac{2 R\left(\pi-\theta^{\mathrm{Y}}\right)}{D}+\frac{2 R\left(\theta^{\mathrm{Y}}-\pi / 2+\gamma\right)}{D}+\frac{2 R_{\text {sag, eff }} \sin \varphi}{D}
$$

where angle $\varphi$ is calculated through equation (25). The liquid-air area fraction is equal to:

$$
f_{\mathrm{la}}=\frac{\text { actual nonwetted area }}{\text { total projected area }}=\frac{2 R_{\text {sag,eff }}}{D}(\beta-\varphi)
$$

Angle $\beta$ is calculated with the help of the same expression included in equation (23).

\subsubsection{Sinusoidal Topography}

Here, the consideration of a curved liquid-air interface due to sagging led to the distinction of three different cases depending on whether there was partial or full wetting. These cases are represented in figure 4. The first criterion for the distinction of cases was based on whether we had partial or full wetting. The y-coordinate of point $M$ [figure 4(a)] determined whether the liquid touched the base of the surface or not. The origin was set at point $O$. If the y-coordinate of point M was positive, we had partial wetting [figures 4(a) and 4(b)]. In the opposite case, full wetting set in [figure 4(c)]. The mathematical condition to distinguish between partial and full wetting is given by equation (29) (see supplementary material):

$$
R_{\text {sag }}\left(1-\sqrt{1-\left(\frac{x_{1}}{R_{\text {sag }}}\right)^{2}}\right)<A\left(1-\cos \left(k x_{1}\right)\right)
$$

where $x_{1}$ is the $\mathrm{X}$-coordinate of point $\mathrm{A}_{1}$ (see figure 4) and was calculated by equation (S7) of the supplementary material. The remaining parameters were the same as those listed in Table 2 and mentioned in Section 2.1.3. The length of the sagging height $h$ determined how far the liquid penetrated the surface asperities. We determined whether we have one $\left(A_{1}\right)$ or two contact points $\left(\mathrm{A}_{1}\right.$ and $\left.\mathrm{N}_{1}\right)$, by solving equation (30) for $\xi$. 
$\left(\cos \xi-\cos \xi_{1}\right)-\frac{R_{\mathrm{sag}}}{A}\left[\sqrt{1-\left(\frac{\xi}{k R_{\mathrm{sag}}}\right)^{2}}-\sqrt{1-\left(\frac{\xi_{1}}{k R_{\mathrm{sag}}}\right)^{2}}\right]=0$

where $\xi=k x$. The roots of equation (30) were the $\xi$-coordinates of points $\mathrm{A}_{1}$ and $\mathrm{N}_{1}$. It is obvious that the coordinate $\xi_{1}$, corresponding to point $\mathrm{A}_{1}$, was a solution to equation (30). If there were two intersection points, the second solution $\xi_{2}$ corresponded to the coordinate of point $\mathrm{N}_{\gamma}$.

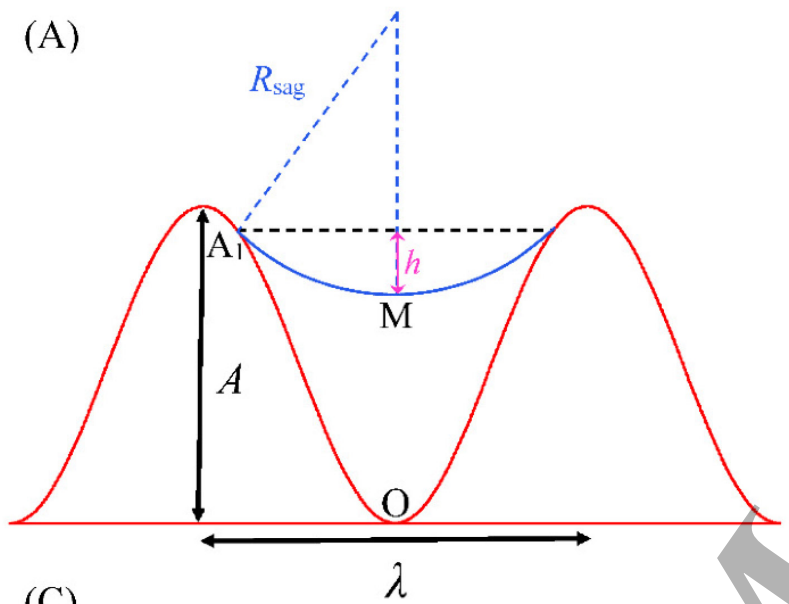

(C)

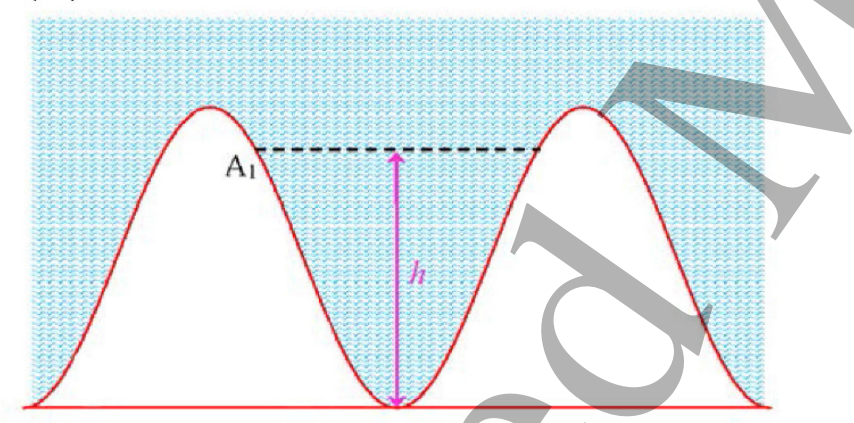

(B)

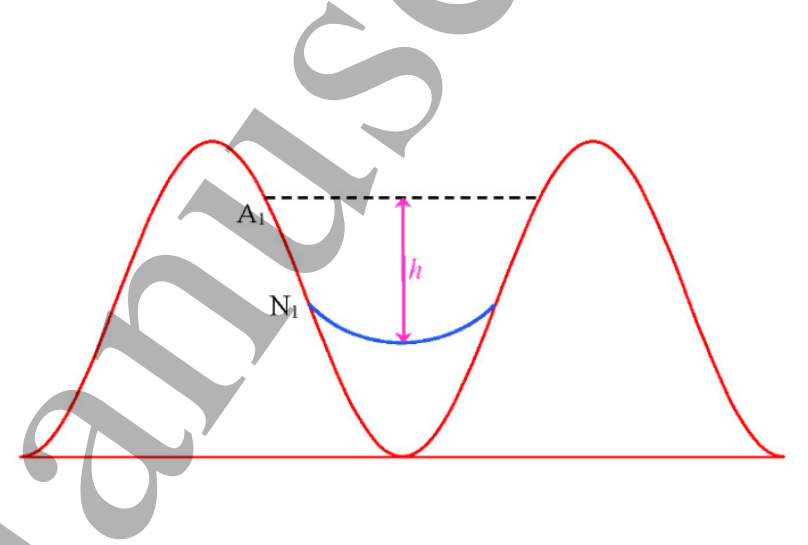

Figure 4. Schematic representation of the three cases considered for the sinusoidal topography: A) partial wetting with one contact point $\left.\left(\mathrm{A}_{1}\right), \mathrm{B}\right)$ partial wetting with two contact points $\left(\mathrm{A}_{1}\right.$ and $\mathrm{N}_{1}$ ) and $\mathrm{C}$ ) full wetting. In all parts, the surface is shown in red. In parts (A) and (B), the liquid interface is depicted with a continuous blue line, whereas in part (C) the liquid is illustrated with

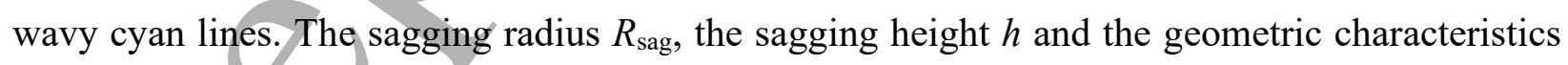
$(A, \lambda)$ of the sinusoidal topography are shown in part (A) (see text for details).

\subsubsection{Partial Wetting with One Contact Point}


This case is represented schematically in figure 4(a). For this case, the solid-liquid area fraction was the same as the one calculated through equation (7) in Section 2.1.3. The liquid-air interface, on the other hand, changed because of the curved shape of the interface considered now. The refined expression for the liquid-air interface is given in equation (31).

$$
f_{\mathrm{la}}=\frac{\text { actual nonwetted area }}{\text { total projected area }}=\frac{2 \beta R_{\mathrm{sag}}}{\lambda}
$$

where $\beta=\arcsin \left(\frac{x_{1}}{R_{\mathrm{sag}}}\right)$.

\subsubsection{Partial Wetting with Two Contact Points}

This case is shown schematically in figure 4(b). The solid-liquid area fraction was calculated through equation (32), which is very similar to equation (7). However, in this case, $x_{2}$ (the $\mathrm{x}$ coordinate of point $\mathrm{N}_{1}$ ) was used. For the calculation of $x_{2}$, the reader is referred to the supplementary material.

$$
f_{\mathrm{sl}}=\left\{\begin{array}{l}
\frac{1}{\pi} \sqrt{1+(A k)^{2}}\left[E\left(\frac{A k}{\sqrt{1+(A k)^{2}}}\right)-E\left(\frac{A k}{\sqrt{1+(A k)^{2}}}, k x_{2}-\frac{\pi}{2}\right)\right], x_{2}>\frac{\lambda}{4} \\
\frac{1}{\pi} \sqrt{1+(A k)^{2}}\left[E\left(\frac{A k}{\sqrt{1+(A k)^{2}}}\right)+E\left(\frac{A k}{\sqrt{1+(A k)^{2}}}, k x_{2}-\frac{\pi}{2}\right)\right], x_{2}<\frac{\lambda}{4}
\end{array}\right.
$$

The liquid-air interface was calculated with the help of equation (31). However, in this case, $x_{2}$ was used for the calculation of angle $\beta$ through: $\beta=\arcsin \left(\frac{x_{2}}{R_{\text {sag }}}\right)$.

\subsubsection{Full Wetting}

In the case of full wetting, the solid-liquid area fraction $f_{\text {sl }}$ was equal to the roughness $r$ of the profile and the liquid-air area fraction $f_{\text {la }}$ was zero. For a regular sinusoidal profile, the roughness $r$ was equal to:

$$
f_{\mathrm{sl}}=\text { roughness }=\frac{2}{\pi} \sqrt{1+(A k)^{2}} E\left(\frac{A k}{\sqrt{1+(A k)^{2}}}\right)
$$


where $E(m)$ denotes the complete elliptic integral of the second kind. Interestingly, the roughness depends only on the ratio of the amplitude to the wavelength.

\subsection{Multiscale Hierarchical Roughness}

In this section, we consider multiscale hierarchical roughness. A generic algorithm was derived for modeling multiple roughness scales to quantify the impact of hierarchical/structure on wettability. However, because most nonwetting biological surfaces comprised two distinct roughness scales, here, up to two levels of roughness were considered. Nonetheless, the derived formalism is applicable to any multiscale hierarchical surface of arbitrarily large multiplicity $n$. Figure 5 shows a few examples of hierarchical double-scale surface topographies. It can be proven (see supplementary material) that the formulas predicting the solid-liquid and liquid-air area fractions for any multiscale topography of multiplicity $n$ are given by equations (34) and (35), respectively. The derivation of the double scale (or, in general, the multiscale) formalism is merely a superposition of the formulas of the individual single scales. Therein, one must consider the expressions of the area fractions of the upper (finer) scale as weights (fractions) acting on the expressions of the lower (coarser) scale. In that regard, the contact point of the supernatant liquid with the upper (finer) scale is determined based on the formalism of this particular topography as described in section 2.2 .

$f_{\mathrm{sl}}\left(\right.$ profile $_{1}, \ldots$, profile $\left._{n}\right)=\prod_{i=1}^{n} f_{\mathrm{sl}}\left(a_{i}, l_{i}\right.$, profile type $_{\mathrm{i}}$, liquid type $)$

$f_{\text {la }}\left(\right.$ profile $_{1}, \ldots$, profile $\left._{n}\right)=\sum_{i=1}^{n}\left[f_{\text {la }}\left(a_{i}, l_{i}\right.\right.$, profile type $_{\mathrm{i}}$, liquid type $) \times$

$$
\left.\prod_{m=1}^{i-1} f_{\mathrm{sl}}\left(a_{n-m}, l_{n-m}, \text { profile type }_{m}, \text { liquid type }\right)\right]
$$


(A)
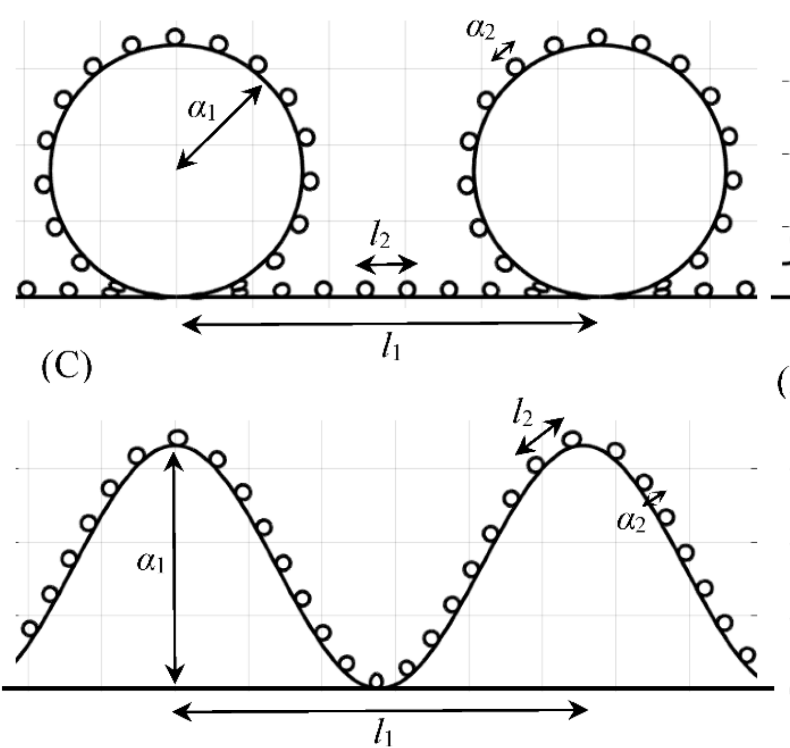

(E)
(B)

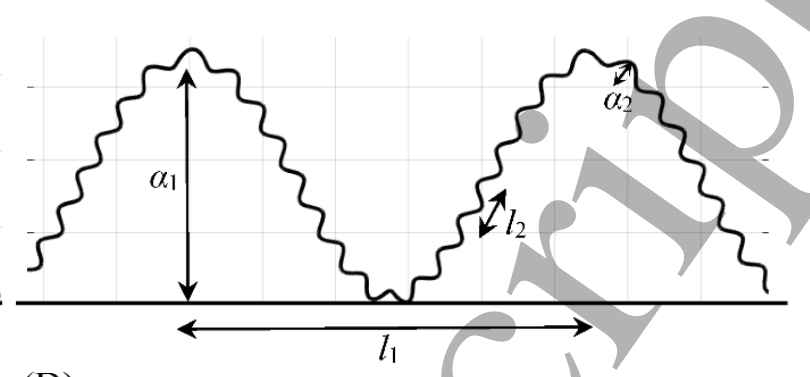

(D)

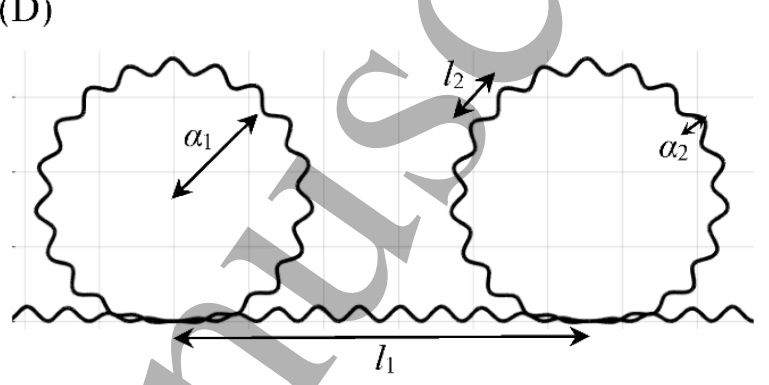

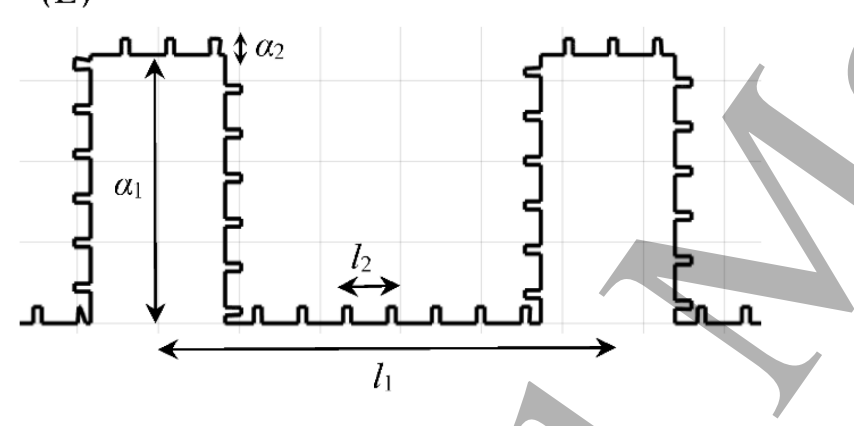

Figure 5. Schematic representations of representative double-scale surface topographies. A) fibers on fibers, B) sinusoid on sinusoid, C) fibers on sinusoid, D) sinusoid on fibers and E) pillars on pillars. In all parts, the topography parameters (see Table 1) describing each length scale are shown. Subscript "1" describes the coarser scale, whereas subscript " 2 " refers to the finer scale.

\section{RESULTS AND DISCUSSION}

\subsection{Single-scale Results}

Figures 6-8 depict heat maps showing the calculated contact angles as a function of the geometrical parameters of pillar, fiber and sinusoid topographies, respectively. To facilitate comparison between the classical and the improved CB models, parts (A-C) in all three figures show the results of the classical CB model for each wetting liquid, whereas parts (D-F) show the results of our model for the same length scale, indicated as SC1, for all three liquids. Parts (G-I) 
show the calculated contact angles based on our model for a much smaller length scale $\mathrm{SC} 2<<$ $\mathrm{SC} 1$ for all three liquids considered here.

Several interesting features can be observed from the heat maps of figures 6-8, Interestingly, in figure 6(a), we observe that the classical CB model predicted pillar surfaces which are phobic to GLY $\left(\mathrm{CA}>90^{\circ}\right)$ for all considered dimensions. This was also true for WAT and DIM drops, as illustrated in figures 6(b) and 6(c), respectively. This observation is misleading and constitutes a first indication of the incapacity of the classical CB model to predict wetting accurately. Furthermore, at large pillar distance $D$ and small pillar width $W$, roughness decreases, and thus the pillar topography becomes progressively a smooth, flat surface. As discussed in Section 1, the contact angle decreases with decreasing roughness and the surface becomes less resistant to wetting. However, figures 6(a-c) show that the classical CB model predicted for all three wetting liquids increasing contact angles with decreasing roughness.

By further reducing the roughness, e.g., by moving the pillars far away from one another and reducing their width, the contact angle is expected to converge to the Young contact angle $\theta^{\mathrm{Y}}$ which is equal to $76^{\circ}$ for GLY, $73^{\circ}$ for WAT and $57^{\circ}$ for DIM on LLDPE (see Table 2). This anticipation was not respected by the classical CB model, which predicted superomniphobicity (contact angle about $160^{\circ}$ ) for this almost-flat surface! For the fiber topography, the trend was similar, and the same misleading results for the classical CB model were obtained. This behavior is depicted in figures 7(a-c) for GLY, WAT and DIM, respectively. Similarly, the same response of the classical CB model, albeit less pronounced, was observed in the case of the sinusoid topography illustrated in figures 8(a-c) for GLY, WAT and DIM, respectively.

In figure 6(d), we show the results of the improved CB model for GLY and pillar topography. First, we observed that the overall behavior was completely different from the one of figure 6(a). The predicted CAs in part (D) were much smaller (almost by a factor of two) than their counterparts predicted by the classical CB model. Second, in sharp contrast to what the classical CB model predicted, here the CA increased with increasing roughness (i.e., as $D$ became smaller and $W$ remained small). As discussed in Section 1, this behavior is in accordance with numerous experimental observations and theoretical predictions asserting that increased roughness led to increased surface-to-volume ratio and thus greater repellency. Third, the improved CB model captured successfully the transition to the Young state. This is evident in figure 6(d), where the predicted contact angle converged to the Young contact angle of GLY $\theta^{\mathrm{Y}}=76^{\circ}$ as $D$ became large 
and the pillars were moved further away from one another. The same trend was observed in the CA behavior of GLY for the remaining two surface topographies illustrated in figures $7(d)$ and 8(d) for fiber and sinusoid topography, respectively.

The same behavior was observed for WAT as wetting liquid, as shown in figures 6(e), 7(e) and 8(e) for pillar, fiber and sinusoid topography, respectively. For the least polar DIM, a similar behavior was observed as figures 6(f), 7(f) and 8(f) show for pillar, fiber and sinusoid topography, respectively. At this point, a clarification is in order. For surfaces with $\theta^{\mathrm{Y}}<90^{\circ}$, there can be no stable CB state. Only a metastable one can exist and only in the case of re-entrant geometries; this implies that, of the three considered single-scale textures, only the "fiber" case can have a metastable CB state. Therefore, we conclude that the transitions observed in figures $7(\mathrm{~d}-\mathrm{f})$ for the fiber topography when going in the direction of reduced roughness were transitions from a metastable CB state to the Wenzel and eventually to the Young state. Contrary to that, the transitions observed in figures $6(\mathrm{~d}-\mathrm{f})$ and $8(\mathrm{~d}-\mathrm{f})$ when moving in the direction of reduced roughness, for pillars and sinusoid topographies respectively, were transitions from a metastable Wenzel to the Young state.

By comparing parts (D), (E) and (F) of figure 6 with their counterparts in figures 7 and 8 , it becomes clear that, for the considered length scale SC1, the fiber topography exhibited the highest CAs, directly followed by the sinusoid topography. The flat-top pillars topography was the least useful in terms of achieving high contact angles and thereby reducing wettability. This observation is valid for all three liquids considered here and is in agreement with previous studies claiming that, in nature, increased contact angles[46] and energetically stable interfaces[75] are associated with surface textures containing curved, round-top features rather than sharp-edged structures. Taken all together, the original CB model led to an overprediction of the CA by $20-35 \%$ depending on wetting liquid and surface topography. We believe that this discrepancy is, in large part, due to the intrinsic assumptions of the classical CB model pertaining to a straight liquid-air interface.

Parts (G-I) of figures 6-8 show the predictions of the improved model at a much smaller length scale $\mathrm{SC} 2<<\mathrm{SC} 1$, by one order of magnitude, for the three topographies and the three wetting liquids. By comparing parts (D-F) with parts (G-I) in every one of these figures, it becomes clear that the CAs at the smaller length scale SC2 were consistently larger than their counterparts at the larger length scale SC1. This observation is in qualitative agreement with previous modeling predictions and experimental observations claiming that "smaller is better" for producing 
omniphobic surfaces.[50] For all three single-scale surface topographies and for all three wetting liquids, the highest CAs were observed in regions characterized by increased nanoscale roughness. For instance, in the case of GLY and fiber topography shown in figure 7(g), the maximum CA was observed at small fiber distance $D$ and small fiber radius $R$. Similarly, for GLY and sinusoid topography, shown in figure $8(\mathrm{~g})$, the high-CA area was located at small wavelengths $\lambda$ and large amplitudes $A$ where roughness was more pronounced.

In agreement with the behavior observed at the large length scale SC1, the fiber profile exhibited the highest CAs also at the smaller length scale SC2. However, in contrast to the SC1 results, the pillar topography surpassed the sinusoid topography at SC2. This behavior can be attributed to the fact that for the pillar topography we assumed that the liquid-air interface was immobilized at the corner points of the pillars. Therefore, the liquid was not allowed to insert the free space between neighboring pillars, which would have led to increased wetting and smaller CAs. The maximal CAs predicted by our model for all three single-scale topographies and all three wetting liquids are summarized in Table 3 . The values of the geometric parameters describing the corresponding surface textures are also listed.

Most importantly, figures 6-8 and Table 3 show that the wetting behavior of all three liquids on any of the surface topographies considered here was qualitatively similar. Consideration of a nonpolar, low-surface-tension liquid (DIM) resulted in a qualitatively analogous wetting behavior for single-scale topographies. This is evident by the similar shape of the CA surfaces calculated for DIM and the other two polar liquids (cf. neighboring columns in figures 6-8). The overall trend remained the same without large deviations in the CA magnitude for all three liquids and for all single-scale surface topographies. Consequently, very similar CAs arose when the three liquids were placed on the same topography type having comparable dimensions. This observation favors the creation of omniphobic surfaces. 

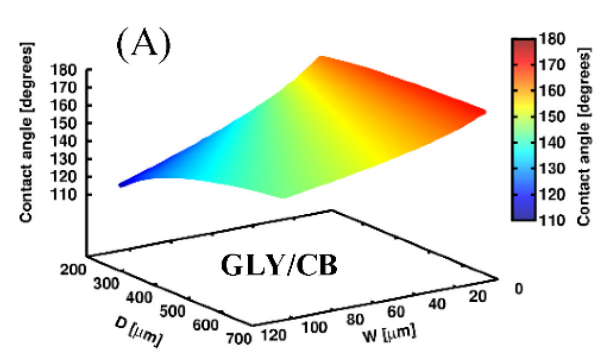

(B)
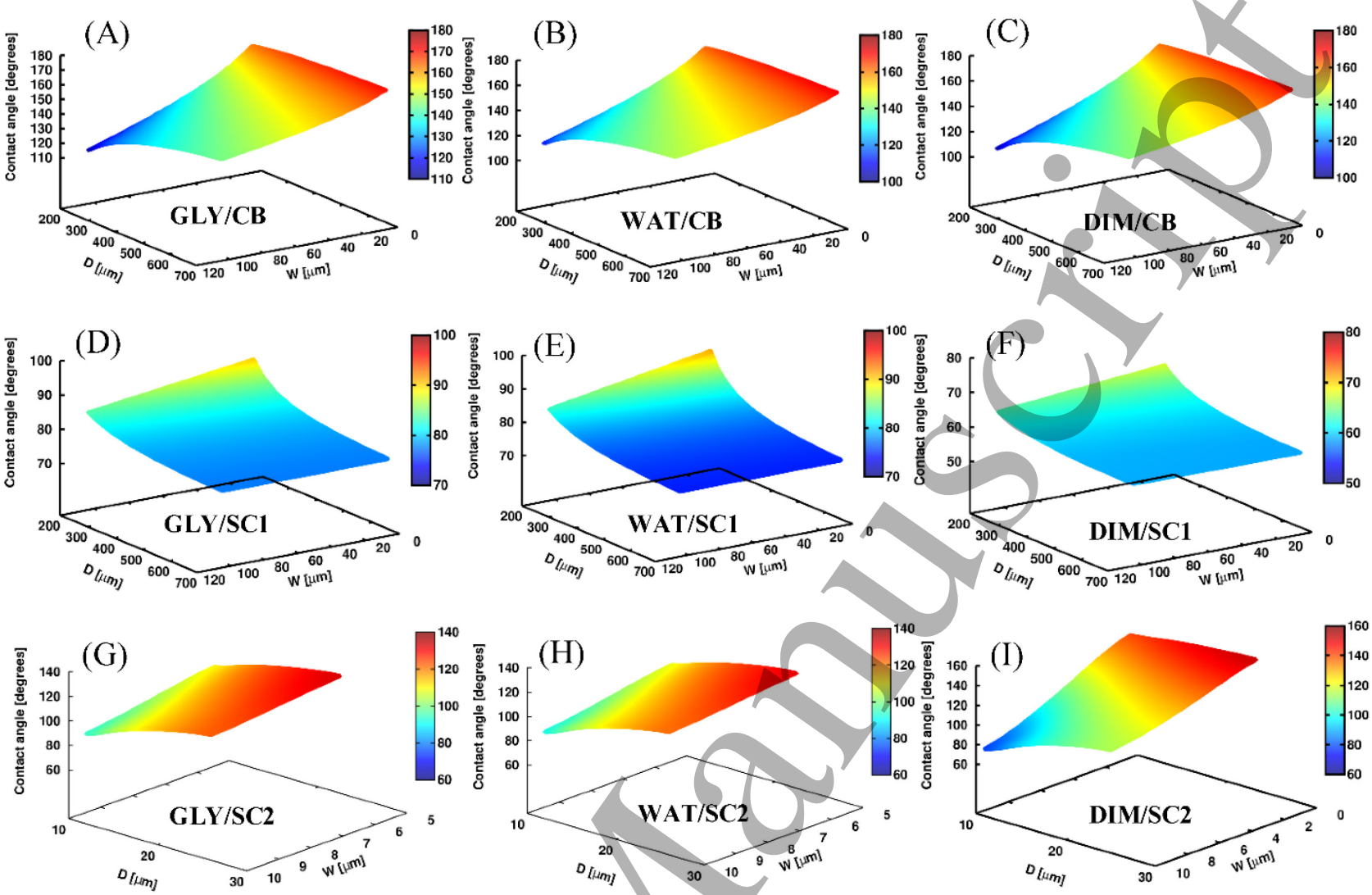

Figure 6. Heat maps of the calculated contact angle for the pillar topography. Rows of this figure correspond to model/length scales (row1: $\mathrm{CB} / \mathrm{SC} 1$, row2: this work/SC1 and row3: this work/SC2) and columns correspond to wetting liquids (column1: GLY, column2: WAT and column3: DIM). Parts (A-C): the classical CB model at SC1, parts (D-F) our model at SC1 and parts (G-I) our model at SC2 $<<\mathrm{SC} 1$. The pillar height $H$ in parts (A-F) was equal to $7 \mu \mathrm{m}$, whereas in parts (GI) equal to $2 \mu \mathrm{m}$. 

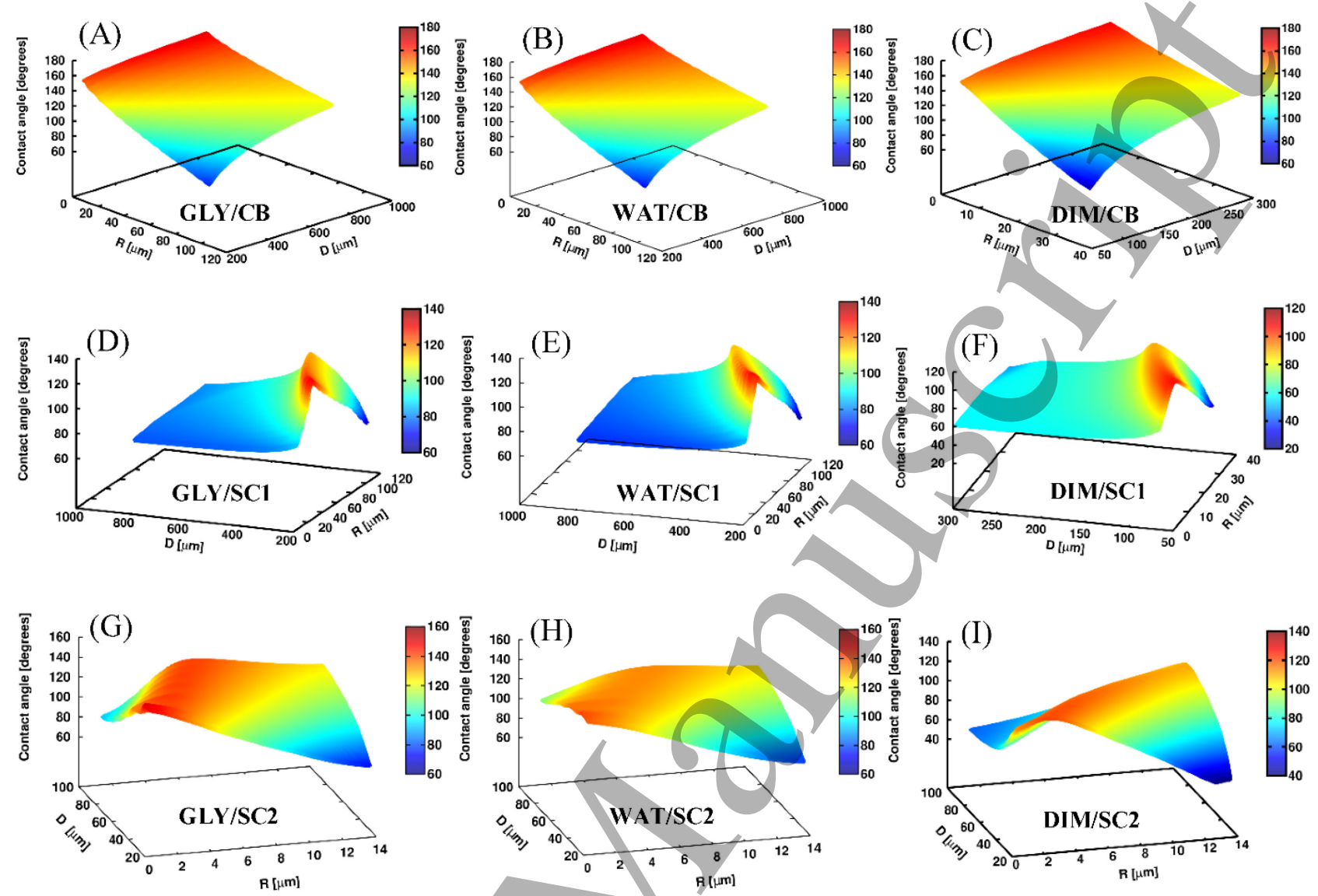

Figure 7. Heat maps of the calculated contact angle for the fiber topography. Rows of this figure correspond to model/length scales (row1: $\mathrm{CB} / \mathrm{SC} 1$, row2: this work/SC1 and row3: this work/SC2) and columns correspond to wetting liquids (column1: GLY, column2: WAT and column3: DIM). Parts (A-C): the classical CB model at SC1, parts (D-F) our model at SC1 and parts (G-I) our model at $\mathrm{SC} 2<<\mathrm{SC} 1$. 

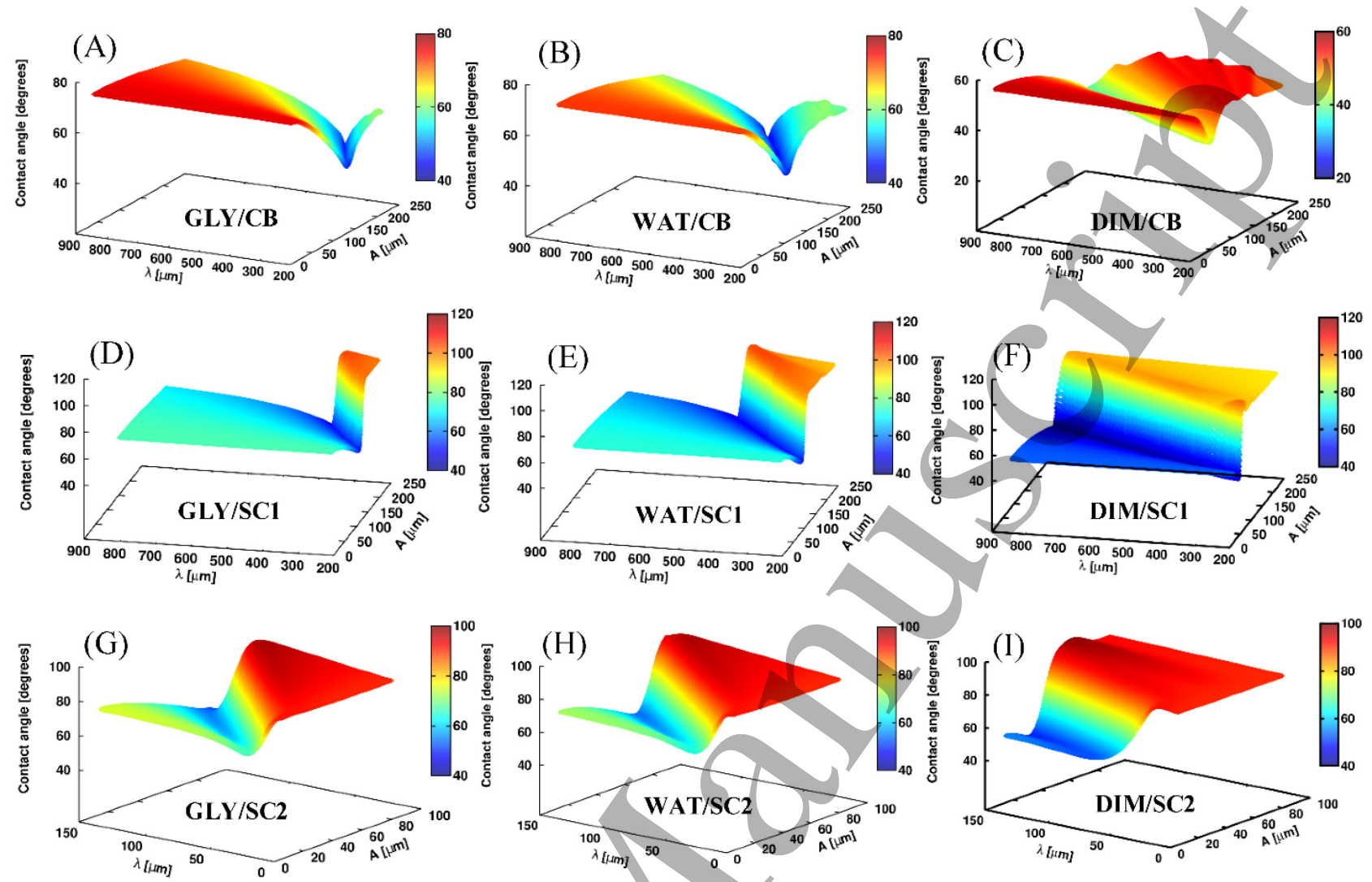

Figure 8. Heat maps of the calculated contact angle for the sinusoid topography. Rows of this figure correspond to model/length scales (row1: $\mathrm{CB} / \mathrm{SC} 1$, row2: this work/SC1 and row3: this work/SC2) and columns correspond to wetting liquids (column1: GLY, column2: WAT and column3: DIM). Parts (A-C): the classical CB model at SC1, parts (D-F) our model at SC1 and parts (G-I) our model at SC2 $<<$ SC1.

Table 3. Maximal predicted contact angles based on the improved CB model for the three singlescale surface topographies and the three wetting liquids considered in this work.

\begin{tabular}{|c|c|c|c|c|c|c|c|c|c|}
\hline $\begin{array}{c}\text { Single-scale } \\
\text { topography }\end{array}$ & \multicolumn{3}{|c|}{ Pillars } & \multicolumn{3}{c|}{ Fibers } & \multicolumn{3}{c|}{ Sinusoid } \\
\hline $\begin{array}{c}\text { Wetting } \\
\text { liquid }\end{array}$ & GLY & WAT & DIM & GLY & WAT & DIM & GLY & WAT & DIM \\
\hline Max. CA [0] & 136 & 135 & 144 & 144 & 142 & 139 & 100 & 99 & 101 \\
\hline $\boldsymbol{\alpha}[\boldsymbol{\mu m}]$ & 6 & 6 & 3 & 3 & 3 & 2 & 81 & 73 & 54 \\
\hline $\boldsymbol{l}[\boldsymbol{\mu m}]$ & 26 & 26 & 25 & 42 & 39 & 34 & 126 & 140 & 140 \\
\hline
\end{tabular}




\subsection{Double-scale Results}

Table 4 summarizes the maximal CAs calculated for the three most relevant double-scale surface topographies and the three wetting liquids. For brevity, the remaining double-scale topographies were omitted from Table 4 because they exhibited CAs smaller than $150^{\circ}$ and were therefore deemed less meaningful for the construction of superomniphobic surfaces. The associated values of the geometric parameters of each length scale and topography are also listed. The first noticeable remark is that double-scale hierarchical roughness led to increased CAs compared to the single-scale roughness. This observation is valid for all three liquids studied here and complies fully with the general literature consensus associating increased roughness (hierarchical or not) with increased resistance to wetting. Contrary to the cases of WAT and GLY, the improvement in the wetting resistance for DIM brought about through consideration of doublescale topographies was somewhat mitigated. This becomes clear by juxtaposing the CA results for DIM listed in Tables 3 and 4 for single- and double-scale topographies, respectively. This behavior was attributed to the relatively greater tendency of DIM to spread and wet the surface owed to its substantially smaller surface tension compared to WAT and GLY.

From Table 4, it becomes clear that double-scale roughness was most efficient when the two length scales differed from each other by at least one order of magnitude. Of the three most relevant double-scale surface topographies presented in Table 4, the "fibers on sine" topography exhibited maximal contact angles over $150^{\circ}$ for all three liquids. Interestingly, this most-promising doublescale topography yielded superphobic CAs also for DIM, which would not have been achieved otherwise for this low-surface-tension liquid. The three wetting liquids showed similar behaviors for all double-scale topographies. Moreover, as Table 4 shows, the associated surface parameters were very close. As in the case of single-scale topographies, this fact facilitates and promotes the construction of superomniphobic surfaces.

Table 4. Maximal predicted contact angle based on the improved CB model for the most relevant double-scale surface topographies and the three wetting liquids considered in this work. The geometrical surface parameters for both scales are also shown. 


\section{CONCLUSIONS}

In summary, we report the development and application of a refined version of the classical CB model for the prediction of surface topographies exhibiting superomniphobic behavior. This was accomplished by considering realistic curved liquid-air interfaces and by incorporating into the classical $\mathrm{CB}$ model the sagging height and sagging radius, which depend only on the physicochemical properties of the wetting liquid and the surface texture. In our analysis, we have neglected the droplet size and weight, while we assumed spherical sessile drops. Our model is based on the original $\mathrm{CB}$ model and is therefore functional in the validity area of the original $\mathrm{CB}$ model, i.e., uniformly rough surfaces with surface heterogeneities larger than atomic or molecular dimensions. This fact mitigates by no means the applicability of the proposed model, as the creation of surface irregularities below the nanometer scale remains a formidable technological task.

The original CB model predicted for all considered liquids phobic surfaces irrespective of the dimensions of the surface topography. Furthermore, it failed to capture the trend between CA and roughness, predicting $\mathrm{CA}$-increase with diminishing roughness. Because of this erroneous trend, the original CB model did not converge to the Young contact angle with reducing roughness. In contrast, our model rendered a more realistic representation of the CA behavior. First, the predicted CAs were smaller than their counterparts predicted by the classical CB model. Overall, direct comparison between the classical $\mathrm{CB}$ model and our model led to a $\mathrm{CA}$ overprediction by the former by $20-35 \%$ depending on wetting liquid and surface topography. Second, the proposed 


\section{SUPPLEMENTARY MATERIAL}

See the supplementary material for details on the derivation of the expressions for the area fractions for the classical and the improved CB models, as well as a generic method to calculate the area fractions for multiscale hierarchical surface topographies. 


\section{ACKNOWLEDGEMENT}

NL, AJ and RK gratefully acknowledge the University of Applied Sciences Western Switzerland (HES-SO) and the College of Engineering and Architecture Fribourg (HEIA-FR) for financial support.

\section{DATA AVAILABILITY STATEMENT}

The data that supports the findings of this study are available from the corresponding author upon reasonable request.

\section{REFERENCES}

[1] Adhikari B, Howes T, Bhandari B R and Truong V/2001 STICKINESS IN FOODS: A REVIEW OF MECHANISMS AND TEST METHODS International Journal of Food Properties 4 1-33

[2] Lai C C 1986 Food Product-Package Compatibility Proceedings, ed J I Gray, Harte, B. R., Miltz, J. (Michigan State University: East Lansing: CRC Press) pp 258-69

[3] Michalski M C, Desobry S and Hardy 1997 Food materials adhesion: A review Critical Reviews in Food Science and Nutrition 37 591-619

[4] Criado M T 1994 The importance of bacterial adhesion in the dairy industry Food Technol. 48 1236

[5] Allen K W 1993 Current theories of adhesion and their relevance to adhesive technology J. Phys. IV France 03 C7-1511-C7-6

[6] Allen K W 1993 Some reflections on contemporary views of theories of adhesion International Journal of Adhesion and Adhesives 13 67-72

[7] Li D and Neumann A W 1992 equation of state for interfacial tensions of solid-liquid systems Advances in Colloid and Interface Science 39 299-345 
[8] Derjaguin B and Landau L 1993 Theory of the stability of strongly charged lyophobic sols and of the adhesion of strongly charged particles in solutions of electrolytes Progress in Surface Science $4330-59$

[9] James A M 1991 Microbial Cell Surface Analysis: Structural and Physicochemical Methods, ed N Mozes, Handley, P. S., Busscher, H. J., Rouxhet, P. G. (New York: VCH Publishers) pp 221-62

[10] Kinloch A J 1980 The science of adhesion Journal of Materials Science 15 2141-66

[11] Nosonovsky M and Bhushan B 2008 Roughness-induced superhydrophobicity: a way to design non-adhesive surfaces Journal of Physics: Condensed Matter 20225009

[12] Young T 1805 III. An essay on the cohesion of fluids Philosophical Transactions of the Royal Society of London 95 65-87

[13] Marmur A and Bittoun E 2009 When Wénzel and Cassie Are Right: Reconciling Local and Global Considerations Langmuir 25 1277-81

[14] Bittoun E and Marmur A 2010 Chemical Nano-Heterogeneities Detection by Contact Angle Hysteresis: Theoretical Feasibility Langmuir 26 15933-7

[15] Mittal K L 2009 Contact Angle, Wettability and Adhesion: Taylor \& Francis)

[16] Goddard J M and Hotchkiss J H 2007 Polymer surface modification for the attachment of bioactive compounds Progress in Polymer Science 32 698-725

[17] Choi W, Tuteja A, Mabry J M, Cohen R E and McKinley G H 2009 A modified Cassie-Baxter relationship to explain contact angle hysteresis and anisotropy on non-wetting textured surfaces Journal of Colloid and Interface Science 339 208-16

[18] Bico J, Thiele $U$ and Quéré D 2002 Wetting of textured surfaces Colloids and Surfaces A: Physicochemical and Engineering Aspects 206 41-6

[19] Wenzel R N 1936 RESISTANCE OF SOLID SURFACES TO WETTING BY WATER Industrial \& Engineering Chemistry 28 988-94 
[20] Cassie A B D and Baxter S 1944 Wettability of porous surfaces Transactions of the Faraday Society $40546-51$

[21] Epstein A K, Pokroy B, Seminara A and Aizenberg J 2011 Bacterial biofilm shows persistent resistance to liquid wetting and gas penetration Proceedings of the National Academy of Sciences 108995

[22] Sun T, Wang G, Feng L, Liu B, Ma Y, Jiang L and Zhu D 2003 Reversible Switching between Superhydrophilicity and Superhydrophobicity Angewandte Chemie International Edition 43 35760

[23] Zhang J, Lu X, Huang W and Han Y 2005 Reversible Superhydrophobicity to Superhydrophilicity Transition by Extending and Unloading an Elastic Polyamide Film Macromolecular Rapid Communications 26 477-80

[24] Lu X, Zhang C and Han Y 2004 Low-Density Polyethylene Superhydrophobic Surface by Control of Its Crystallization Behavior Macromolecular Rapid Communications 25 1606-10

[25] Seemann R, Brinkmann M, Herminghaus S, Khare K, Law B M, McBride S, Kostourou K, Gurevich E, Bommer S, Herrmann C and Michler D 2011 Wetting morphologies and their transitions in grooved substrates Journal of Physics: Condensed Matter 23184108

[26] Khare K, Brinkmann M, Law B M, Herminghaus S and Seemann R 2009 Switching wetting morphologies in triangular grooves The European Physical Journal Special Topics 166 151-4

[27] Nakajima A, Fujishima A, Hashimoto K and Watanabe T 1999 Preparation of Transparent Superhydrophobic Boehmite and Silica Films by Sublimation of Aluminum Acetylacetonate Advanced Materials 11 1365-8

[28] Chen W, Fadeev A Y, Hsieh M C, Öner D, Youngblood J and McCarthy T J 1999 Ultrahydrophobic and Ultralyophobic Surfaces: Some Comments and Examples Langmuir 15 3395-9 
[29] Sun T, Song W and Jiang L 2005 Control over the responsive wettability of poly(Nisopropylacrylamide) film in a large extent by introducing an irresponsive molecule Chemical Communications $1723-5$

[30] Pichon A 2009 Repelling oil Nature Chemistry

[31] Chhatre S S, Guardado J O, Moore B M, Haddad T S, Mabry J M, McKinley G H and Cohen R E 2010 Fluoroalkylated Silicon-Containing Surfaces-Estimation of Solid-Surface Energy ACS Applied Materials \& Interfaces 2 3544-54

[32] Nishino T, Meguro M, Nakamae K, Matsushita M and Ueda Y 1999 The Lowest Surface Free Energy Based on -CF3 Alignment Langmuir 15 4321-3

[33] Kijlstra J, Reihs K and Klamt A 2002 Roughness and topology of ultra-hydrophobic surfaces Colloids and Surfaces A: Physicochemical and Engineering Aspects 206 521-9

[34] Zhang X, Shi F, Yu X, Liu H, Fu Y, Wang Z, Jiang L and Li X 2004 Polyelectrolyte Multilayer as Matrix for Electrochemical Deposition of Gold Clusters: Toward Super-Hydrophobic Surface Journal of the American Chemical Society 126 3064-5

[35] Xie Q, Xu J, Feng L, Jiang L, Tang W, Luo X and Han C C 2004 Facile Creation of a Super-Amphiphobic Coating Surface with Bionic Microstructure Advanced Materials 16 302-5

[36] Shiu J-Y, Kuo C-W, Chen P and Mou C-Y 2004 Fabrication of Tunable Superhydrophobic Surfaces by Nanosphere Lithography Chemistry of Materials 16 561-4

[37] Youngblood J P and McCarthy T J 1999 Ultrahydrophobic Polymer Surfaces Prepared by Simultaneous Ablation of Polypropylene and Sputtering of Poly(tetrafluoroethylene) Using Radio Frequency Plasma Macromolecules 32 6800-6

[38] Woodward I, Schofield W C E, Roucoules V and Badyal J P S 2003 Super-hydrophobic Surfaces Produced by Plasma Fluorination of Polybutadiene Films Langmuir 19 3432-8 
[39] Feng L, Li S, Li H, Zhai J, Song Y, Jiang L and Zhu D 2002 Super-Hydrophobic Surface of Aligned Polyacrylonitrile Nanofibers Angewandte Chemie International Edition 41 1221-3

[40] Tuteja A, Choi W, Ma M, Mabry J M, Mazzella S A, Rutledge G C, McKinley G H and Cohen RE 2007 Designing Superoleophobic Surfaces Science 3181618

[41] Tuteja A, Choi W, Mabry J M, McKinley G H and Cohen R E 2008 Robust omniphobic surfaces Proceedings of the National Academy of Sciences 10518200

[42] Herminghaus S 2000 Roughness-induced non-wetting EPL (Europhysics Letters) 52165

[43] Cheng Y-T and Rodak D E 2005 Is the lotus leaf superhydrophobic? Applied Physics Letters 86 144101

[44] Marmur A 2003 Wetting on Hydrophobic Rough Surfaces: To Be Heterogeneous or Not To Be? Langmuir 19 8343-8

[45] Nosonovsky M 2007 Multiscale Roughness and Stability of Superhydrophobic Biomimetic Interfaces Langmuir 23 3157-61

[46] Bittoun E and Marmur A 2012 The Role of Multiscale Roughness in the Lotus Effect: Is It Essential for Super-Hydrophobicity? Langmuir 28 13933-42

[47] Nosonovsky M and Bhushan B 2008 Biologically Inspired Surfaces: Broadening the Scope of Roughness** Advanced Functional Materials 18 843-55

[48] Onda T, Shibuichi S, Satoh N and Tsujii K 1996 Super-Water-Repellent Fractal Surfaces Langmuir $122125-7$

[49] Zhai L, Cebeci F Ç, Cohen R E and Rubner M F 2004 Stable Superhydrophobic Coatings from Polyelectrolyte Multilayers Nano Letters 4 1349-53

[50] Chhatre S S, Choi W, Tuteja A, Park K-C, Mabry J M, McKinley G H and Cohen R E 2010 Scale Dependence of Omniphobic Mesh Surfaces Langmuir 26 4027-35 
[51] Gao L and McCarthy T J 2006 The "Lotus Effect" Explained: Two Reasons Why Two Length Scales of Topography Are Important Langmuir 22 2966-7

[52] Verho T, Bower C, Andrew P, Franssila S, Ikkala O and Ras R H A 2010 Mechanically Durable Superhydrophobic Surfaces Advanced Materials 23 673-8

[53] Ma M and Hill R M 2006 Superhydrophobic surfaces Current Opinion in Colloid \& Interface Science 11 193-202

[54] Barbieri L, Wagner E and Hoffmann P 2007 Water Wetting Transition Parameters of Perfluorinated Substrates with Periodically Distributed Flat-Top Microscale Obstacles Langmuir 23 1723-34

[55] Kang S M, You I, Cho W K, Shon H K, Lee T G, Choi I S, Karp J M and Lee H 2010 One-Step Modification of Superhydrophobic Surfaces by a Mussel-Inspired Polymer Coating Angewandte Chemie International Edition $499401-4$

[56] Chauhan P, Kumar A and Bhushan B 2019 Self-cleaning, stain-resistant and anti-bacterial superhydrophobic cotton fabric prepared by simple immersion technique Journal of Colloid and Interface Science 535 66-74

[57] Li F, Bhushan B, Pan Y and Zhao X 2019 Bioinspired superoleophobic/superhydrophilic functionalized cotton for efficient separation of immiscible oil-water mixtures and oil-water emulsions Journal of Colloid and Interface Science 548 123-30

[58] Nanda D, Sahoo A, Kumar A and Bhushan B 2019 Facile approach to develop durable and reusable superhydrophobic/superoleophilic coatings for steel mesh surfaces Journal of Colloid and Interface Science 535 50:7

[59] Gurera D and Bhushan B 2018 Fabrication of bioinspired superliquiphobic synthetic leather with self-cleaning and low adhesion Colloids and Surfaces A: Physicochemical and Engineering Aspects

$545130-7$ 
[60] Bhushan B 2019 Bioinspired materials and surfaces for green science and technology Philosophical Transactions of the Royal Society A: Mathematical, Physical and Engineering Sciences 377 20180336

[61] Bhushan B and Martin S 2018 Substrate-independent superliquiphobic coatings for water, oil, and surfactant repellency: An overview Journal of Colloid and Interface Science 526 90-105

[62] Höcker H 2002 Plasma treatment of textile fibers Pure and Applied Chemistry 74 423-7

[63] Zimmermann J, Reifler F A, Fortunato G, Gerhardt L-C and Seeger S 2008 A Simple, One-Step Approach to Durable and Robust Superhydrophobic Textiles Advanced Functional Materials 18 $3662-9$

[64] Gao X, Yan X, Yao X, Xu L, Zhang K, Zhang J, Yang B and Jiang L 2007 The Dry-Style Antifogging Properties of Mosquito Compound Eyes and Artificial Analogues Prepared by Soft Lithography Advanced Materials 19 2213-7

[65] Cao L, Jones A K, Sikka V K, Wu J and Gao D 2009 Anti-Icing Superhydrophobic Coatings Langmuir 25 12444-8

[66] Pan Q and Wang M 2009 Miniature Boats with Striking Loading Capacity Fabricated from Superhydrophobic Copper Meshes ACS Applied Materials \& Interfaces 1 420-3

[67] Ou J, Perot B and Rothstein J P 2004 Laminar drag reduction in microchannels using ultrahydrophobic surfaces Physics of Fluids 16 4635-43

[68] Marmur A 2006 Underwater Superhydrophobicity: Theoretical Feasibility Langmuir 22 1400-2

[69] Gurera D and Bhushan B 2019 Optimization of bioinspired conical surfaces for water collection from fog Journal of Colloid and Interface Science 551 26-38

[70] Wilson SJ and Hutley M C 1982 The Optical Properties of 'Moth Eye' Antireflection Surfaces Optica Acta: International Journal of Optics 29 993-1009 
[71] Arzt E, Gorb S and Spolenak R 2003 From micro to nano contacts in biological attachment devices Proceedings of the National Academy of Sciences 10010603

[72] Andreas S, Zdenek C, Boris F S, Manuel S and Wilhelm B 2007 The dream of staying clean: Lotus and biomimetic surfaces Bioinspiration \& Biomimetics 2 S126

[73] Gao X and Jiang L 2004 Water-repellent legs of water striders Nature 43236

[74] Bushnell D M and Moore K J 1991 Drag Reduction in Nature Annual Review of Fluid Mechanics 23 $65-79$

[75] Nosonovsky M and Bhushan B 2009 Superhydrophobic surfaces and emerging applications: Nonadhesion, energy, green engineering Current Opinion in Colloid \& Interface Science 14 270-80

[76] Afferrante L and Carbone G 2010 Microstructured superhydrorepellent surfaces: effect of drop pressure on fakir-state stability and apparent contact angles Journal of Physics: Condensed Matter 22325107

[77] Afferrante L and Carbone G 2014 The effect of drop volume and micropillar shape on the apparent contact angle of ordered microstructured surfaces Soft Matter 10 3906-14

[78] Afferrante L and Carbone G 2015 Statistical theory of wetting of liquid drops on superhydrophobic randomly rough surfaces Physical Review E 92042407

[79] Ciavarella M and Afferrante L 2016 Adhesion of rigid rough contacts with bounded distribution of heights Tribology International 100 18-23

[80] Jung M, Brinkmann M, Seemann R, Hiller T, Sanchez de La Lama M and Herminghaus S 2016 Wettability controls slow immiscible displacement through local interfacial instabilities Physical Review Fluids 1074202

[81] Tretyakov N, Papadopoulos P, Vollmer D, Butt H-J, Dünweg B and Daoulas K C 2016 The CassieWenzel transition of fluids on nanostructured substrates: Macroscopic force balance versus microscopic density-functional theory The Journal of Chemical Physics 145134703 
[82] Violano G and Afferrante L 2019 Contact of rough surfaces: Modeling adhesion in advanced multiasperity models Proceedings of the Institution of Mechanical Engineers, Part J: Journal of Engineering Tribology 233 1585-93

[83] Bhushan B 2016 Biomimetics: Bioinspired Hierarchical-Structured Surfaces for Green Science and Technology: Springer International Publishing)

[84] Nosonovsky M and Bhushan B 2007 Hierarchical roughness optimization for biomimetic superhydrophobic surfaces Ultramicroscopy 107 969-79

[85] Nosonovsky M and Bhushan B 2008 Multiscale Dissipative Mechanisms and Hierarchical Surfaces: Friction, Superhydrophobicity, and Biomimetics: Springer Berlin Heidelberg)

[86] Nosonovsky M and Bhushan B 2008 Patterned Nonadhesive Surfaces: Superhydrophobicity and Wetting Regime Transitions Langmuir 24 1525-33

[87] Dufour R, Semprebon C and Herminghaus S 2016 Filling transitions on rough surfaces: Inadequacy of Gaussian surface models Physical Review E 93032802

[88] Herminghaus S 2012 Universal Phase Diagram for Wetting on Mesoscale Roughness Physical Review Letters 109236102

[89] Pu X, Li G and Huang H 2016 Preparation, anti-biofouling and drag-reduction properties of a biomimetic shark skin surface Biology Open 5389

[90] Koch K, Bhushan B, Jung Y C and Barthlott W 2009 Fabrication of artificial Lotus leaves and significance of hierarchical structure for superhydrophobicity and low adhesion Soft Matter $\mathbf{5}$ $1386-93$

[91] Kwon Y, Patankar N, Choi J and Lee J 2009 Design of Surface Hierarchy for Extreme Hydrophobicity Langmuir 25 6129-36

[92] Chibowski E and Jurak M 2013 Comparison of contact angle hysteresis of different probe liquids on the same solid surface Colloid and Polymer Science 291 391-9 
[93] Wang Y, Sang D K, Du Z, Zhang C, Tian M and Mi J 2014 Interfacial Structures, Surface Tensions, and Contact Angles of Diiodomethane on Fluorinated Polymers The Journal of Physical Chemistry

\section{118 10143-52}

[94] Carbone G and Mangialardi L 2005 Hydrophobic properties of a wavy rough substrate The European Physical Journal E 16 67-76

[95] Bottiglione F and Carbone G 2013 Role of Statistical Properties of Randomly Rough Surfaces in Controlling Superhydrophobicity Langmuir 29 599-609

[96] Brown P S and Bhushan B 2015 Designing bioinspired superoleophobic surfaces APL Materials 4 015703

[97] Brown P S and Bhushan B 2016 Durable, superoleophobic polymer-nanoparticle composite surfaces with re-entrant geometry via solvent-induced phase transformation Scientific Reports 6 21048

[98] Batchelor G K 2000 An Introduction to Fluid Dynamics (Cambridge: Cambridge University Press)

[99] Marmur A 2004 The Lotus Effect: Superhydrophobicity and Metastability Langmuir 20 3517-9

[100] Bittoun E and Marmur A 2009 Optimizing Super-Hydrophobic Surfaces: Criteria for Comparison of Surface Topographies Journal of Adhesion Science and Technology 23 401-11

[101] Lampropoulos N K, Dimakopoulos Y and Tsamopoulos J 2016 Transient flow of gravity-driven viscous films over substrates with rectangular topographical features Microfluidics and Nanofluidics 2051

[102] Karapetsas G, Lampropoulos N K, Dimakopoulos Y and Tsamopoulos J 2017 Transient flow of gravity-driven viscous films over 3D patterned substrates: conditions leading to Wenzel, Cassie and intermediate states Microfluidics and Nanofluidics 2117

[103] Varchanis S, Dimakopoulos Y and Tsamopoulos J 2017 Steady film flow over a substrate with rectangular trenches forming air inclusions Physical Review Fluids 2124001 
[104] Pettas D, Karapetsas G, Dimakopoulos Y and Tsamopoulos J 2017 On the degree of wetting of a slit by a liquid film flowing along an inclined plane Journal of Fluid Mechanics 820 5-41

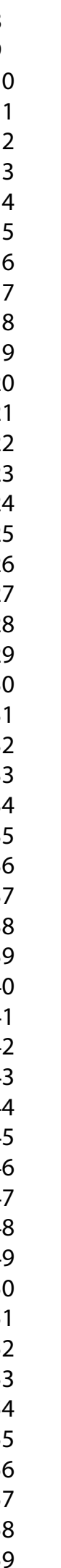

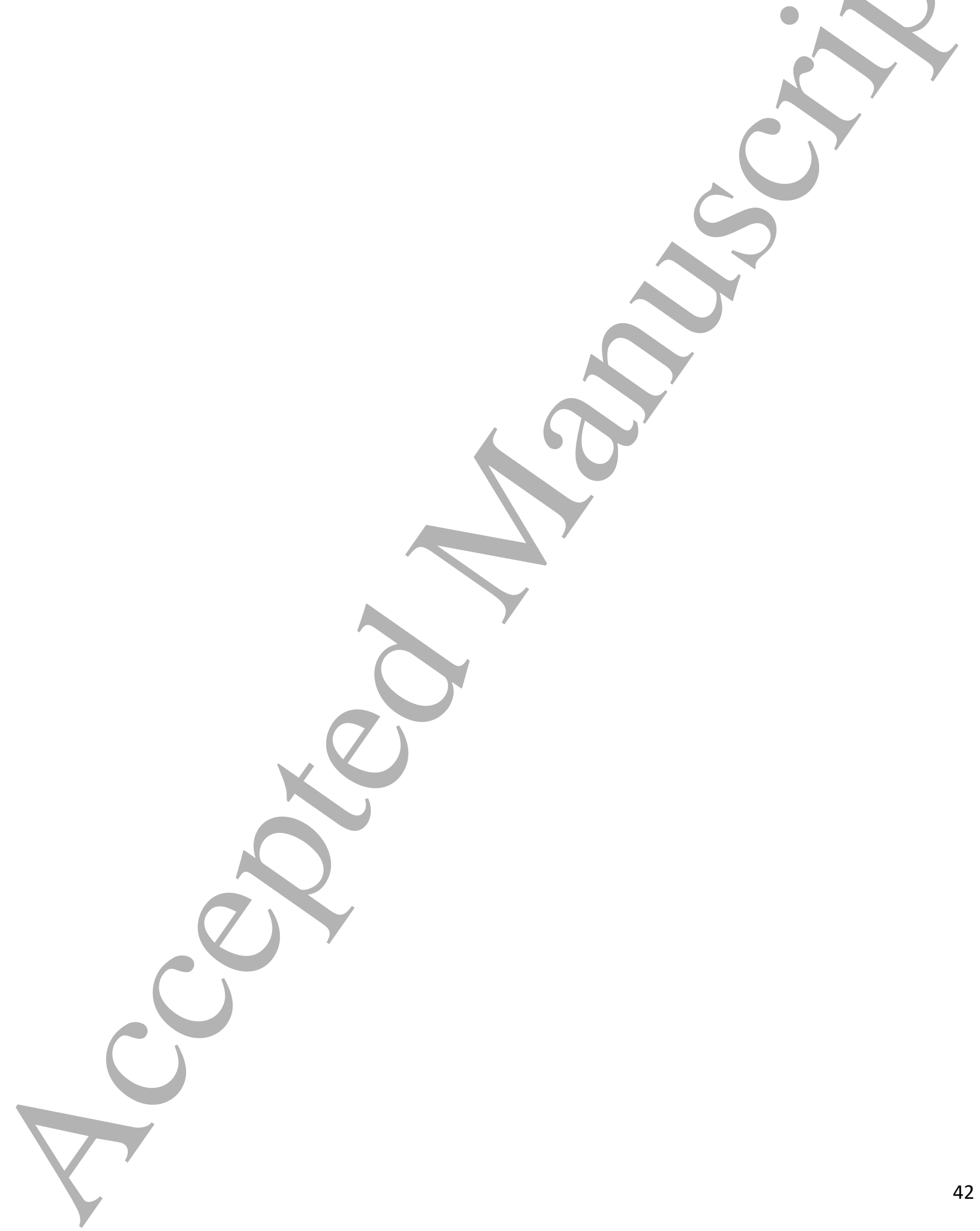

